\begin{tabular}{|c|l|}
\hline Title & A characterization of coactions whose fixed-point algebras contain special maximal abelian *-subalgebras \\
\hline Author(s) & A oi, Hisashi; Y amanouchi, Takehiko \\
\hline Citation & $\begin{array}{l}\text { Ergodic Theory and Dynamical Systems, 26(6), 1673-1706 } \\
\text { https://doi.org/10.1017/S014338570600459 }\end{array}$ \\
\hline Issue Date & 2006 \\
\hline Doc URL & http://hdl.handle.net/2115/18641 \\
\hline Rights & Copyright $\odot 2006$ Cambridge University Press \\
\hline Type & article \\
\hline File Information & download.pdf \\
\hline
\end{tabular}

Instructions for use 


\title{
A characterization of coactions whose fixed-point algebras contain special maximal abelian *-subalgebras
}

\author{
HISASHI AOI $\dagger$ and TAKEHIKO YAMANOUCHI \\ Department of Mathematics, Faculty of Science, Hokkaido University, \\ Sapporo 060-0810, Japan \\ (e-mail: $\{$ aoi, yamanouc\}@math.sci.hokudai.ac.jp)
}

(Received 14 January 2005 and accepted in revised form 21 June 2006)

\begin{abstract}
It is shown that, for the von Neumann algebra $A$ obtained from a principal measured groupoid $\mathcal{R}$ with the diagonal subalgebra $D$ of $A$, there exists a natural 'bijective' correspondence between coactions on $A$ that fix $D$ pointwise and Borel 1-cocycles on $\mathcal{R}$. As an application of this result, we classify a certain type of coactions on approximately finite-dimensional type II factors up to cocycle conjugacy. By using our characterization of coactions mentioned above, we are also able to generalize to some extent those results of Zimmer concerning 1-cocycles on ergodic equivalence relations into compact groups.
\end{abstract}

\section{Introduction}

For each action $\alpha$ of a locally compact quantum group $\mathbb{G}=(M, \Delta)$ on a von Neumann algebra $A$, we obtain an inclusion of $A$ and the fixed-point algebra $A^{\alpha}$. The Galois theory for $\alpha$ means the natural correspondence between the intermediate subalgebras of $\left(A^{\alpha} \subseteq A\right)$ and the left coideals of $\mathbb{G}$. It is known that there exists a bijective Galois correspondence when $\mathbb{G}$ is a compact Kac algebra and the action $\alpha$ on a factor $A$ is minimal, i.e. the relative commutant $\left(A^{\alpha}\right)^{\prime} \cap A$ is trivial [17]. In particular, if $\alpha$ is a minimal action of an ordinary compact group $K$, then there exists a bijective correspondence between the set of intermediate subalgebras of $\left(A^{\alpha} \subseteq A\right)$ and the set of closed subgroups of $K$ (we note that the minimality of $\alpha$ implies that all the intermediate subalgebras of $\left(A^{\alpha} \subseteq A\right.$ ) are factors). Motivated by this work, many results concerning minimal actions have been obtained $[\mathbf{6}, \mathbf{2 9}, \mathbf{3 6}]$. However, there seems to be little hope that the arguments made in those results go beyond the case of minimal actions.

$\dagger$ Current address: Department of Mathematics, Faculty of Science and Technology, Sophia University, Tokyo 102-8854, Japan (e-mail: aoi@mm.sophia.ac.jp). 
In the meantime, by the recent work of the first author, it turns out that there exists a sort of Galois correspondence for an inclusion of a von Neumann algebra (which is not necessarily a factor) and a Cartan subalgebra. Let $A$ be a (separable) von Neumann algebra and $D$ be a maximal abelian von Neumann subalgebra of $A$. The subalgebra $D$ is called a Cartan subalgebra of $A$ if $D$ is regular in $A$ and is the range of a faithful normal conditional expectation from $A$ onto $D$. It is well known that, if $A$ has a Cartan subalgebra $D$, then there exists a discrete measured equivalence relation $\mathcal{R}$ on a standard Borel space $(X, \mathfrak{B}, \mu)$ such that $(D \subseteq A)$ is isomorphic to $\left(W^{*}(X) \subseteq W^{*}(\mathcal{R}, \sigma)\right)$, where $W^{*}(\mathcal{R}, \sigma)$ is the (twisted) groupoid von Neumann algebra of $\mathcal{R}$ and $W^{*}(X)$ is the diagonal algebra of $W^{*}(\mathcal{R}, \sigma)$ isomorphic to $L^{\infty}(X)$. Under this situation, the main result of [2] states that there exists a bijective correspondence between the set of subrelations and that of intermediate subalgebras:

$$
\begin{aligned}
& B \mapsto \mathcal{S}_{B} \subseteq \mathcal{R}, \\
& \mathcal{S} \mapsto W^{*}\left(\mathcal{S},\left.\sigma\right|_{\mathcal{S}}\right) \subseteq A .
\end{aligned}
$$

We emphasize that the above correspondence contains all intermediate von Neumann subalgebras of $D \subseteq A$ which are not necessarily factors.

On the other hand, the authors studied quantum group actions on von Neumann algebras whose fixed-point algebras contain Cartan subalgebras. They showed that, if a finitedimensional Kac algebra $\mathbb{K}$ admits a minimal action $\alpha$ on a factor $A$ such that $A^{\alpha}$ contains a Cartan subalgebra $D$ of $A$, then $\mathbb{K}$ is necessarily cocommutative [3, Theorem 4.1]. To prove this theorem, they used the fact that $A^{\alpha}$ was determined by some equivalence subrelation. However, thanks to the result of [2] mentioned above, this is always the case as long as the action $\alpha$ fixes the Cartan subalgebra. So, if it is only the fact that $A^{\alpha}$ is described by a subrelation that is needed for their result, then we can hopefully expect to eliminate the following two assumptions in the above theorem: (i) the finite-dimensionality of $\mathbb{K}$; (ii) the minimality of $\alpha$ (in particular, the factoriality of $A$ ). In other words, it is natural to ask whether the cocommutativity of a possibly infinite-dimensional quantum group follows if it admits a (not necessarily minimal) action on a von Neumann algebra that fixes a Cartan subalgebra pointwise.

The first aim of this paper is to give a complete answer to this question under a more general situation. Namely, we will show that if $A$ has a regular maximal abelian subalgebra $D$, and if $\alpha: A \rightarrow M \otimes A$ is a faithful action of a locally compact quantum group $\mathbb{G}=(M, \Delta)$ on $A$ such that $A^{\alpha}$ contains $D$, then $\mathbb{G}$ must be cocommutative (Corollary 3.2).

The idea used to prove this claim is as follows: since $D$ is a maximal abelian *-subalgebra, for each element $v$ of the normalizer of $D$ in $A, w:=\alpha(v)\left(1 \otimes v^{*}\right)$ belongs to $M \otimes\left(D^{\prime} \cap A\right)=M \otimes D$. So $w$ is an $M$-valued function. An easy computation shows that $\left(\Delta \otimes \operatorname{id}_{A}\right)(w)=w_{12} w_{23}$, i.e. $w$ is an $I G(\mathbb{G})$-valued function, where $\operatorname{IG}(\mathbb{G})$ is the intrinsic group of $\mathbb{G}$ (see Proposition 3.1). So we have that $\alpha(v)=w(1 \otimes v$ ) belongs to $I G(\mathbb{G})^{\prime \prime} \otimes A$. Since $D$ is regular in $A$, this means that $\alpha$ is a coaction.

Thus, the study of quantum group actions with the property described above can be reduced to that of coactions of locally compact groups. 
The second aim of this paper is to give a characterization of such coactions (i.e. coactions that fix regular maximal abelian $*$-subalgebras). One finds without difficulty that every 1-cocycle on a (principal) measured groupoid into a group $K$ gives rise to a coaction of $K$ on the corresponding groupoid von Neumann algebra, which fixes the (regular) diagonal subalgebra. Conversely, we will prove that any coaction on the groupoid von Neumann algebra with this property arises in this way (Theorem 5.8). Therefore, this result roughly establishes a 'bijective' correspondence between the 1-cocycles on a principal measured groupoid and the coactions on the associated groupoid von Neumann algebra whose fixed-point algebras contain the regular diagonal subalgebra. The 1-cocycles on measured equivalence relations are well-studied objects in ergodic theory. So, in this paper, we will begin analyzing the coactions of the type described above in terms of 1-cocycles by fully utilizing this useful correspondence.

As the first consequence of the analysis along this line, we will prove that two coactions $\alpha$ and $\alpha^{\prime}$ of the above type are cocycle conjugate to each other if the corresponding 1-cocycles $c_{\alpha}$ and $c_{\alpha^{\prime}}$ are cohomologous to each other (Proposition 4.8). Conversely, we will show that if $\alpha$ is cocycle conjugate to $\alpha^{\prime}$, and if the automorphism that gives this cocycle conjugacy leaves the diagonal subalgebra globally invariant, then $c_{\alpha}$ is weakly equivalent to $c_{\alpha^{\prime}}$ (Proposition 5.10). We also show that the Connes spectrum of a coaction coincides with the asymptotic range of the corresponding 1-cocycle (Theorem 6.3). This, together with one of the results in [14] concerning the 1-cocycles on amenable ergodic equivalence relations, enables us to extend the main result in [19] to general coactions on the AFD (approximately finite-dimensional) type II factors. More precisely, we will prove the uniqueness, up to cocycle conjugacy, of coactions of a locally compact group on the AFD type II factor with full Connes spectrum fixing a Cartan subalgebra pointwise.

Moreover, our study generalizes the results of Zimmer concerning 1-cocycles on ergodic equivalence relations into compact groups. In [37, Theorem 3.5], Zimmer proved that a 1-cocycle $c$ is cohomologous to a 1-cocycle $c^{\prime}$ into a closed subgroup $H$ of $K$ if and only if the Mackey action of $c$ is $K$-isomorphic to the transitive space $K / H$. We succeed in generalizing this result for all 1-cocycles $c$ on (not necessarily ergodic) discrete measured equivalence relations into general locally compact groups. Indeed, we will prove that a 1-cocycle $c$ into $K$ is cohomologous to a 1-cocycle $c^{\prime}$ into a subgroup $H$ of $K$ if and only if the Mackey action of $c$ is an extension of the homogeneous $K$-space $K / H$, which is also equivalent to the condition that the dual action $\widehat{\alpha_{c}}$ is induced by some action of $H$ (Theorem 7.2). By using this result, we will introduce the notion of regularity of a 1-cocycle.

The organization of the paper is as follows. In $\S 2$, we summarize the basic facts about measured groupoids and group coactions. In $\S 3$, we will prove that, for a locally compact quantum group $\mathbb{G}$, the existence of an action of $\mathbb{G}$ whose fixed-point algebra contains a special masa implies that $\mathbb{G}$ is cocommutative. In $\S 4$, we prove that each Borel 1-cocycle $c$ on a measured groupoid $\mathcal{G}$ determines a coaction $\alpha_{c}$ on the groupoid von Neumann algebra $A$ of $\mathcal{G}$ so that its fixed-point algebra contains the diagonal subalgebra. The converse to this statement is shown in $\$ 5$ when $\mathcal{G}$ is an equivalence relation. Namely, we will show that, for every coaction $\alpha$ on $A$ the fixed-point algebra of which contains the diagonal subalgebra, there exists a 1-cocycle $c$ 
such that $\alpha$ is equal to $\alpha_{c}$. In $\S 6$, we prove that the Connes spectrum of a coaction of the type described above can be phrased in terms of the corresponding 1-cocycle. In §7, we give a complete answer to the problem as to when a 1-cocycle $c: \mathcal{R} \rightarrow K$ is cohomologous to a 1-cocycle $c^{\prime}: \mathcal{R} \rightarrow K$ whose range is contained in a closed subgroup of $K$. The main tool to attack this problem is the theory of induced actions due to Takesaki [31]. Since, in this paper, we adopt a definition of induced actions slightly different from (but still equivalent to) the original definition given in [31], we include our definition of induced actions in Appendix A as for the readers' convenience. We conclude this paper with the results about integrability of coactions treated above. In $\S 8$, we prove that a coaction $\alpha$ of a locally compact group $K$ that comes from a 1 -cocycle is integrable if and only if $K$ is discrete, provided that the von Neumann algebra that $K$ coacts is determined by a discrete equivalence relation. In this case, it is known that there exists a canonical surjective $*$-homomorphism $\rho$ from the crossed product $\widehat{K}_{\alpha} \ltimes A$ onto the basic extension of $A^{\alpha} \subseteq A$. We also give a necessary and sufficient condition for $\rho$ being faithful.

\section{Definitions and notation}

In this section, we summarize the basic facts about measured groupoids and von Neumann algebras associated to them. Further details regarding these objects can be found in $[\mathbf{8}, \mathbf{1 0}, \mathbf{1 5}, \mathbf{3 3}, \mathbf{3 7}]$. We also briefly discuss group coactions on von Neumann algebras.

We assume that all von Neumann algebras in this paper have separable preduals, and that all locally compact groups satisfy the second axiom of countability.

For a faithful normal semifinite weight $\phi$ on a von Neumann algebra $A$, we set

$$
\mathfrak{n}_{\phi}:=\left\{x \in A: \phi\left(x^{*} x\right)<\infty\right\}, \quad \mathfrak{m}_{\phi}:=\mathfrak{n}_{\phi}^{*} \mathfrak{n}_{\phi}, \quad \mathfrak{m}_{\phi}^{+}:=\mathfrak{m}_{\phi} \cap A_{+} .
$$

The Hilbert space obtained from $\phi$ by the GNS (Gelfand-Neumark-Segal)-construction will be denoted by $H_{\phi}$, and we let $\Lambda_{\phi}: \mathfrak{n}_{\phi} \rightarrow H_{\phi}$ stand for the natural injection.

For a (separable) Hilbert space $\mathcal{H}$, we let $B(\mathcal{H})$ denote the algebra of all bounded operators on $\mathcal{H}$. The center of a von Neumann algebra $A$ will be denoted by $\mathcal{Z}(A)$.

2.1. Measured groupoids and groupoid von Neumann algebras. Let $\mathcal{G}$ be a standard Borel groupoid. We always assume that all relevant maps and sets that are related to the groupoid structure of $\mathcal{G}$ are Borel. We denote the source (respectively the range) of an element $\gamma \in \mathcal{G}$ by $s(\gamma)$ (respectively $r(\gamma)$ ). The unit space of $\mathcal{G}$, which is the image of $\mathcal{G}$ under the source map or the range map, is denoted by $X$. For every $x \in X$, set $\mathcal{G}^{x}:=r^{-1}(\{x\}), \mathcal{G}_{x}:=s^{-1}(\{x\})$. We assume from now on that this groupoid $\mathcal{G}$ admits a Haar system $\left\{\lambda^{x}\right\}_{x \in X}$ and a quasi-invariant $\sigma$-finite measure $\mu$ on $X$. Let $v$ be the $\sigma$-finite measure on $\mathcal{G}$ given by integrating the Haar system with respect to $\mu: v=\int \lambda^{x} d \mu(x)$. We also let $\lambda_{x}(x \in X)$ and $v^{-1}$ be the images of $\lambda^{x}$ and $\nu$, respectively, under the inverse map $\gamma \mapsto \gamma^{-1}$. By definition, $v$ is equivalent to $v^{-1}$ in the sense of absolute continuity, and we put $\delta:=d \nu / d \nu^{-1}$. Hereafter, we call a standard Borel groupoid with such a system $\left(\mathcal{G},\left\{\lambda^{x}\right\}_{x \in X}, \mu\right)$ a measured groupoid.

Throughout the rest of this subsection, we fix a measured groupoid $\left(\mathcal{G},\left\{\lambda^{x}\right\}_{x \in X}, \mu\right)$. 
Let $\mathcal{G}^{(2)}$ denote the set of all multiplicative pairs of $\mathcal{G}$, i.e. $\mathcal{G}^{(2)}:=\left\{\left(\gamma_{1}, \gamma_{2}\right) \in \mathcal{G} \times \mathcal{G}\right.$ : $\left.s\left(\gamma_{1}\right)=r\left(\gamma_{2}\right)\right\}$. On $\mathcal{G}^{(2)}$, we have a measure $v^{(2)}$ defined by $\int f\left(\gamma_{1}, \gamma_{2}\right) d v^{(2)}\left(\gamma_{1}, \gamma_{2}\right)=$ $\iint f\left(\gamma_{1}, \gamma_{2}\right) d \lambda^{s\left(\gamma_{1}\right)}\left(\gamma_{2}\right) d v^{-1}\left(\gamma_{1}\right)$ (refer to [25]).

A Borel map $c$ from $\mathcal{G}$ into a locally compact group $K$ is called a (Borel) 1-cocycle if it satisfies

$$
c\left(\gamma_{1} \gamma_{2}\right)=c\left(\gamma_{1}\right) c\left(\gamma_{2}\right)
$$

for all $\left(\gamma_{1}, \gamma_{2}\right) \in \mathcal{G}^{(2)}$. If another 1-cocycle $c^{\prime}: \mathcal{G} \rightarrow K$ satisfies the equation

$$
c^{\prime}(\gamma)=\varphi(r(\gamma)) c(\gamma) \varphi(s(\gamma))^{-1} \quad(\nu \text {-almost everywhere })
$$

for some Borel map $\varphi: X \rightarrow K$, then we say that $c^{\prime}$ is cohomologous to $c$. Suppose that $\mathcal{G}_{1}$ and $\mathcal{G}_{2}$ are measured groupoids and $c_{i}: \mathcal{G}_{i} \rightarrow K$ are Borel 1-cocycles. We say that $c_{1}$ and $c_{2}$ are weakly equivalent if there exists a Borel and groupoid isomorphism $\rho$ from $\mathcal{G}_{1}$ onto $\mathcal{G}_{2}$ which also preserves the null sets such that $c_{2} \circ \rho$ is cohomologous to $c_{1}$.

For each Borel 1-cocycle $c: \mathcal{G} \rightarrow K$, we introduce the measured groupoid structure on $\mathcal{G}_{c}:=K \times \mathcal{G}$ by the following process: the set $\mathcal{G}_{c}^{(2)}$ of multiplicative pairs consists of points of the form $\left(\left(k, \gamma_{1}\right),\left(c\left(\gamma_{1}\right)^{-1} k, \gamma_{2}\right)\right)$, where $k \in K$ and $\left(\gamma_{1}, \gamma_{2}\right) \in \mathcal{G}^{(2)}$. The product and the inverse are defined by

$$
\left(k, \gamma_{1}\right)\left(c\left(\gamma_{1}\right)^{-1} k, \gamma_{2}\right):=\left(k, \gamma_{1} \gamma_{2}\right), \quad(k, \gamma)^{-1}:=\left(c(\gamma)^{-1} k, \gamma^{-1}\right) .
$$

So we have $r(k, \gamma)=(k, r(\gamma))$ and $s(k, \gamma)=\left(c(\gamma)^{-1} k, s(\gamma)\right)$. Hence the unit space of $\mathcal{G}_{c}$ is (identified with) $K \times X$. It is easy to see that

$$
\lambda^{(k, x)}=(\text { the point mass at } k) \times \lambda^{x} \quad((k, x) \in K \times X)
$$

is a Haar system, and (left Haar measure) $\times \mu$ is a quasi-invariant measure. The module $\delta_{c}$ is given by $\delta_{c}(k, \gamma)=\delta(\gamma)$. The measured groupoid $\mathcal{G}_{c}$ thus obtained is called the skew-product of $\mathcal{G}$ by $c$ or the kernel of $c$. It is well known (see [8, Proposition 7.2]) that the isomorphism class of the skew-product $\mathcal{G}_{c}$ depends only on the cohomology class of $c$.

A Borel map $\sigma: \mathcal{G}^{(2)} \rightarrow \mathbf{T}$ is called a (Borel) 2-cocycle on $\mathcal{G}$ if it satisfies

$$
\begin{gathered}
\sigma\left(\gamma_{1}, \gamma_{2} \gamma_{3}\right) \sigma\left(\gamma_{2}, \gamma_{3}\right)=\sigma\left(\gamma_{1}, \gamma_{2}\right) \sigma\left(\gamma_{1} \gamma_{2}, \gamma_{3}\right) \quad\left(s\left(\gamma_{1}\right)=r\left(\gamma_{2}\right), s\left(\gamma_{2}\right)=r\left(\gamma_{3}\right)\right) \\
\sigma(r(\gamma), \gamma)=\sigma(\gamma, s(\gamma))=1 \quad(\gamma \in \mathcal{G}) .
\end{gathered}
$$

By using the regular representation of the measured groupoid $\mathcal{G}$, we obtain a von Neumann algebra acting on $L^{2}(\mathcal{G}, v)$. We first define the subspace $\mathfrak{A}_{I}$ of $L^{2}(\mathcal{G}, v)$ by

$$
\mathfrak{A}_{I}:=\left\{\xi \in L^{2}(\mathcal{G}, \nu): \xi \text { is } \delta \text {-bounded and }\|\xi\|_{I}<\infty\right\} .
$$

See [15] and [34] for the definition and properties of $\mathfrak{A}_{I}$ and for the terminology used above. Fix a Borel 2-cocycle $\sigma$ on $\mathcal{G}$. We then introduce a product and an involution on $\mathfrak{A}_{I}$ as follows:

$$
\begin{aligned}
(f * g)(\gamma) & :=\int f\left(\gamma_{1}\right) g\left(\gamma_{1}^{-1} \gamma\right) \sigma\left(\gamma_{1}, \gamma_{1}^{-1} \gamma\right) d \lambda^{r(\gamma)}\left(\gamma_{1}\right), \\
f^{\sharp}(\gamma) & :=\sigma\left(\gamma, \gamma^{-1}\right)^{-1} \delta(\gamma)^{-1} \overline{f\left(\gamma^{-1}\right)} .
\end{aligned}
$$


By the same argument as in [15] and [34], one can show that $\mathfrak{A}_{I}$ is a left Hilbert algebra (in fact, a Tomita algebra) in $L^{2}(\mathcal{G}, v)$. The left von Neumann algebra of $\mathfrak{A}_{I}$ is denoted by $W^{*}(\mathcal{G}, \sigma)$ and is called the groupoid von Neumann algebra of $\mathcal{G}$ (or, more precisely, the groupoid von Neumann algebra of $\left.\left(\mathcal{G},\left\{\lambda^{x}\right\}_{x \in X}, \mu\right)\right)$. The modular operator $\nabla$, the modular conjugation $J$ and the right involution $b$ of $\mathfrak{A}_{I}$ are given by

$$
\begin{gathered}
\nabla \xi:=\delta \xi, \quad\{J \xi\}(\gamma)=\sigma\left(\gamma, \gamma^{-1}\right)^{-1} \delta(\gamma)^{-1 / 2} \overline{\xi\left(\gamma^{-1}\right)} \quad\left(\xi \in \mathfrak{A}_{I}\right), \\
\xi^{b}(\gamma)=\sigma\left(\gamma, \gamma^{-1}\right)^{-1} \overline{\xi\left(\gamma^{-1}\right) .}
\end{gathered}
$$

The left multiplication and the right multiplication of $f \in \mathfrak{A}_{I}$ are respectively denoted by $L^{\sigma}(f)$ and $R^{\sigma}(f)$ :

$$
L^{\sigma}(f) \xi:=f * \xi, \quad R^{\sigma}(f) \xi:=\xi * f \quad\left(\xi \in \mathfrak{A}_{I}\right) .
$$

The abelian von Neumann algebra $L^{\infty}(X, \mu)$ is embedded into $W^{*}(\mathcal{G}, \sigma)$ through the representation $f \in L^{\infty}(X, \mu) \longmapsto f \circ r$. We will always identity $L^{\infty}(X, \mu)$ with its image under this representation. This algebra is called the diagonal subalgebra of $W^{*}(\mathcal{G}, \sigma)$.

If the groupoid $\mathcal{G}$ is principal, then $\mathcal{G}$ is a measured equivalence relation and we denote it by $\mathcal{R}$.

We recall that an abelian von Neumann subalgebra $B$ of a von Neumann algebra $A$ is called a quasi-Cartan subalgebra of $A$ under the following conditions:

(1) the subalgebra $B$ is normal, i.e. $B$ is the center of its centralizer in $A$, and $B^{\prime} \cap A$ is of type I;

(2) the subalgebra $B$ is regular in $A$, i.e. the normalizer $\mathcal{N}_{A}(D)$ of $D$ in $A$ generates $A$, where

$$
\mathcal{N}_{A}(D):=\left\{u \in A: u \text { is unitary and } u D u^{*}=D\right\}
$$

(3) there exists a faithful normal conditional expectation from $A$ onto $B$.

By [8, Theorem 8.8], for each von Neumann algebra $A$ having a regular maximal abelian von Neumann subalgebra $D$ that contains a quasi-Cartan subalgebra $B$ of $A$, there exists a measured equivalence relation $\mathcal{R}$ over a standard Borel probability measure space $(X, \mu)$ and a Borel 2-cocycle $\sigma$ on $\mathcal{R}$ such that $(A \supseteq D)$ is isomorphic to $\left(W^{*}(\mathcal{R}, \sigma) \supseteq\right.$ the diagonal subalgebra $L^{\infty}(X, \mu)$ ). In particular, we know from [10, Theorem 1] that the relation $\mathcal{R}$ is discrete (i.e. each equivalence class is a countable set) precisely when the algebra $D$ above coincides with $B$. In that case, we say that $D$ is a Cartan subalgebra of $A$.

2.2. Group coactions on von Neumann algebras. Let $K$ be a locally compact group. We denote by $W^{*}(K)$ the group von Neumann algebra of $K$, i.e. the von Neumann algebra generated by the left regular representation $\lambda_{K}$ of $K$ on $L^{2}(K)$. Remark that $W^{*}(K)$ is the left von Neumann algebra of the left Hilbert algebra $C_{c}(K)$ of all continuous functions on $K$ with compact support, where we consider on $C_{c}(K)$ the usual convolution and involution. The faithful semifinite normal weight on $W^{*}(K)$ associated with the left Hilbert algebra $C_{c}(K)$ is denoted by $\varphi_{K}$, the Plancherel weight on $W^{*}(K)$. It is well known that the predual $A(K)$ of $W^{*}(K)$ has a structure of a commutative involutive Banach algebra. It is called the Fourier algebra of $K$, and it will be freely identified in this paper with 
a dense $*$-subalgebra of the $\mathrm{C}^{*}$-algebra of all continuous functions on $K$ vanishing at infinity (cf. [7, 23]).

There is a special normal unital $*$-isomorphism $\Delta_{K}$ from $W^{*}(K)$ into $W^{*}(K) \otimes W^{*}(K)$, called the coproduct of $W^{*}(K)$, defined by

$$
\Delta_{K}(x):=W_{K}(1 \otimes x) W_{K}^{*} \quad\left(x \in W^{*}(K)\right),
$$

where $W_{K}$ is a unitary on $L^{2}(K) \otimes L^{2}(K)=L^{2}(K \times K)$ given by $\left\{W_{K} \xi\right\}(g, h):=$ $\xi(h g, h)\left(\xi \in L^{2}(K \times K)\right)$. The coproduct satisfies $\Delta_{K}\left(\lambda_{K}(k)\right)=\lambda_{K}(k) \otimes \lambda_{K}(k)$ for all $k \in K$. It is also known that $\left\{x \in W^{*}(K) \backslash\{0\}: \Delta_{K}(x)=x \otimes x\right\}=\lambda_{K}(K)$.

A coaction of $K$ on a von Neumann algebra $A$ is a normal unital $*$-isomorphism $\alpha$ from $A$ into $W^{*}(K) \otimes A$ satisfying $\left(\Delta_{K} \otimes \mathrm{id}_{A}\right) \circ \alpha=\left(\operatorname{id}_{W^{*}(K)} \otimes \alpha\right) \circ \alpha$.

Suppose that $\alpha$ is a coaction of $K$ on a von Neumann algebra $A$.

(1) The von Neumann subalgebra $A^{\alpha}:=\{a \in A: \alpha(a)=1 \otimes a\}$ is called the fixed-point algebra of $\alpha$.

(2) The map $T_{\alpha}$ defined by $T_{\alpha}(a):=\left(\varphi_{K} \otimes \mathrm{id}_{A}\right)(\alpha(a))$ is an operator valued weight from $A$ to $A^{\alpha}$. The coaction $\alpha$ is said to be integrable if $T_{\alpha}$ is semifinite.

(3) The crossed product of $A$ by $\alpha$ is the von Neumann algebra $\widehat{K}_{\alpha} \ltimes A:=(\alpha(A) \cup$ $\left.L^{\infty}(K) \otimes \mathbf{C}\right)^{\prime \prime}$.

(4) For each $k \in K, \hat{\alpha}_{k}:=\left.\operatorname{Ad}\left(\rho_{K}(k) \otimes 1\right)\right|_{\widehat{K}_{\alpha} \ltimes A}$ defines a $*$-automorphism of $\widehat{K}_{\alpha} \ltimes A$, where $\rho_{K}$ is the right regular representation of $K$. We call $\hat{\alpha}$ the dual action of $\alpha$.

(5) A unitary $V$ in $W^{*}(K) \otimes A$ is called an $\alpha$-1-cocycle if $V$ satisfies the following:

$$
\left(\Delta_{K} \otimes \operatorname{id}_{A}\right)(V)=V_{23}\left(\operatorname{id}_{W^{*}(K)} \otimes \alpha\right)(V) .
$$

For each $\alpha$-1-cocycle $V$, Ad $V \circ \alpha$ is also a coaction of $K$ on $A$. A coaction $\alpha^{\prime}$ of $K$ on $A$ is said to be cocycle conjugate to $\alpha$ if there exist an $\alpha$-1-cocycle $V$ and a *-automorphism $\theta$ of $A$ such that $\left(\operatorname{id}_{W^{*}(K)} \otimes \theta\right) \circ \alpha^{\prime} \circ \theta^{-1}=\operatorname{Ad} V \circ \alpha$.

There is a 'spectral theory' for group coactions on von Neumann algebras. We give a brief review of this theory below. We refer the readers to [21, 22, 23] for rudimentary results on the spectral theory of a coaction.

As before, let $\alpha$ be a coaction of $K$ on a von Neumann algebra $A$.

For any $a \in A$, define

$$
\operatorname{Sp}_{\alpha}(a):=\left\{k \in K: \phi(k)=0\left(\forall \phi \in I_{a}\right)\right\},
$$

where $I_{a}:=\left\{\phi \in A(K):\left(\phi \otimes \operatorname{id}_{A}\right)(\alpha(a))=0\right\}$. For a closed subset $E$ of $K$, we set $A^{\alpha}(E):=\left\{a \in A: \operatorname{Sp}_{\alpha}(a) \subseteq E\right\}$. The spectrum $\operatorname{Sp}(\alpha)$ of $\alpha$ is defined as

$$
\operatorname{Sp}(\alpha)=\overline{\bigcup_{a \in A} \operatorname{Sp}_{\alpha}(a)}=\left\{k \in K: \phi(k)=0\left(\forall \phi \in I_{\alpha}\right)\right\}
$$

where $I_{\alpha}:=\left\{\phi \in A(K):\left(\phi \otimes \mathrm{id}_{A}\right) \circ \alpha=0\right\}$. Finally, the Connes spectrum $\Gamma(\alpha)$ of $\alpha$ is the intersection of all $\mathrm{Sp}\left(\alpha^{e}\right)$, where $e$ runs over all non-zero projections in $A^{\alpha}$. Here, for a projection $e \in A^{\alpha}, \alpha^{e}$ stands for the coaction of $K$ on the reduced von Neumann algebra $e A e$ defined by $\alpha^{e}(b):=\alpha(b)(b \in e A e)$. The Connes spectrum of a coaction is always a closed subgroup (cf. [23, page 66]). 
3. Some reductions to coaction case

This section is concerned with a locally compact quantum group action whose fixed-point algebra contains a regular maximal abelian subalgebra. We show below that the existence of such an action implies that the quantum group is cocommutative. Thus, the study of quantum group actions with this property can be reduced to that of coactions of locally compact groups.

Let $\mathbb{G}=(M, \Delta)$ be a locally compact quantum group (in the sense of Kustermans and Vaes) and $\alpha$ be an action of $\mathbb{G}$ on a von Neumann algebra $A$. For the definitions of a locally compact quantum group and its action on a von Neumann algebra, refer to [20] and [33].

We consider the situation where $A$ contains a von Neumann subalgebra $D$ that satisfies the following conditions:

(1) the subalgebra $D$ is maximal abelian in $A$;

(2) the fixed-point algebra $A^{\alpha}$ contains $D$;

(3) the normalizing groupoid $\mathcal{G N}_{A}(D)$ generates $A$, where

$$
\begin{gathered}
\mathcal{G N}_{A}(D):=\left\{v \in A: v \text { is partial isometry and satisfies } v v^{*}, v^{*} v \in D,\right. \\
\left.v D v^{*}=D v v^{*}\right\} .
\end{gathered}
$$

Proposition 3.1. The von Neumann subalgebra $\left\{\left(\operatorname{id}_{M} \otimes \omega\right)(\alpha(a)): a \in A, \omega \in A_{*}\right\}^{\prime \prime}$ of $M$ is contained in $I G(\mathbb{G})^{\prime \prime}$, where $I G(\mathbb{G}):=\{x \in M \backslash\{0\}: \Delta(x)=x \otimes x\}$.

Proof. We identify $D$ with $L^{\infty}(X, \mu)$, where $X$ is a compact metric space and $\mu$ is a positive Radon probability measure.

Let $v \in \mathcal{G N}_{A}(D)$, and set $w:=\alpha(v)\left(1 \otimes v^{*}\right) \in M \otimes A$. Note that, by assumption, we have $v D v^{*} \subseteq D \subseteq A^{\alpha}$. Hence, for any $b \in D$, we have

$$
w(1 \otimes b)=\alpha(v) \alpha\left(v^{*} b v\right)\left(1 \otimes v^{*}\right)=(1 \otimes b) w .
$$

This shows that $w$ belongs to $M \otimes D$. So, by [32, Theorem 7.17], $w$ may be regarded as an $M$-valued bounded measurable function on $X$. From the above result, it follows that $\left(\operatorname{id}_{M} \otimes \alpha\right)(w)=w_{13}$. So we get

$$
\begin{aligned}
\left(\Delta \otimes \mathrm{id}_{A}\right)(w) & =\left(\Delta \otimes \mathrm{id}_{A}\right)(\alpha(v))\left(1 \otimes 1 \otimes v^{*}\right) \\
& =\left(\operatorname{id}_{M} \otimes \alpha\right)(\alpha(v))\left(1 \otimes 1 \otimes v^{*}\right) \\
& =\left(\operatorname{id}_{M} \otimes \alpha\right)(w(1 \otimes v))\left(1 \otimes 1 \otimes v^{*}\right) \\
& =w_{13} w_{23} .
\end{aligned}
$$

This identity implies that, as a function on $X, w$ satisfies $\Delta(w(x))=w(x) \otimes w(x)$ for almost every $x \in X$. Therefore, we may view $w$ as an $I G(\mathbb{G})$-valued measurable function on $X$. So, for any $\omega \in A_{*}$, we have

$$
\left(\operatorname{id}_{M} \otimes \omega\right)(\alpha(v))=\left(\operatorname{id}_{M} \otimes \omega\right)(w(1 \otimes v))=\left(\operatorname{id}_{M} \otimes v \omega\right)(w) \in I G(\mathbb{G})^{\prime \prime} .
$$

From this and the fact that the linear subspace generated by $\mathcal{G N}_{A}(D)$ is $\sigma$-weakly dense in $A$, it follows that

$$
\begin{aligned}
& \left\{\left(\operatorname{id}_{M} \otimes \omega\right)(\alpha(a)): a \in A, \omega \in A_{*}\right\}^{\prime \prime} \\
& \quad=\left\{\left(\operatorname{id}_{M} \otimes \omega\right)(\alpha(u)): u \in \mathcal{G N}_{A}(D), \omega \in A_{*}\right\}^{\prime \prime} \subseteq I G(\mathbb{G})^{\prime \prime} .
\end{aligned}
$$

Thus we are done. 
A locally compact quantum group $\mathbb{G}=(M, \Delta)$ is said to be cocommutative if the coproduct $\Delta$ satisfies $\sigma \circ \Delta=\Delta$, where $\sigma$ denotes the flip on $M \otimes M$. It is well-known that a locally compact quantum group $\mathbb{G}=(M, \Delta)$ is cocommutative if and only if there exists a unique locally compact group $K$ such that $(M, \Delta)=\left(W^{*}(K), \Delta_{K}\right)$. An action $\alpha$ of a locally compact quantum group $\mathbb{G}=(M, \Delta)$ on a von Neumann algebra $A$ is said to be faithful (cf. $[\mathbf{1 7}, \mathbf{3 3}])$ if $\left\{\left(\operatorname{id}_{M} \otimes \omega\right)(\alpha(a)): a \in A, \omega \in A_{*}\right\}^{\prime \prime}=M$.

COROLlary 3.2. Let $\mathbb{G}=(M, \Delta)$ be a locally compact quantum group. If $\mathbb{G}$ admits a faithful action $\alpha$ on a von Neumann algebra A containing a regular maximal abelian von Neumann subalgebra $D$ inside $A^{\alpha}$, then $\mathbb{G}$ must be cocommutative.

\section{Coactions derived from 1-cocycles}

In this section, we will show that, when a 1-cocycle from a measured groupoid into a locally compact group is given, one can construct a coaction of the group on the associated groupoid von Neumann algebra. We think that this fact might be known to specialists. We were not however able to find the literature that actually contains the proof, except for the case where the group is abelian (see [25, Ch. II, §5]). So we include the proof here for the readers' convenience. This would also help to motivate the discussions made in the following sections.

Throughout this section, we fix a measured groupoid $\left(\mathcal{G},\left\{\lambda^{x}\right\}_{x \in X}, \mu\right)$. Let us consider a Borel 1-cocycle $c$ from $\mathcal{G}$ into a locally compact group $K$. Define a linear operator $U_{c}$ on the Hilbert space $L^{2}(K) \otimes L^{2}(\mathcal{G}, v)$ by

$$
\left\{U_{c} \xi\right\}(k, \gamma):=\xi\left(c(\gamma)^{-1} k, \gamma\right) \quad\left(\xi \in L^{2}(K \times \mathcal{G}), k \in K, \gamma \in \mathcal{G}\right) .
$$

It is easy to see that $U_{c}$ is a unitary.

LEMMA 4.1. The unitary $U_{c}$ defined above belongs to $W^{*}(K) \otimes B\left(L^{2}(\mathcal{G}, v)\right)$. Moreover it is a 1-cocycle of the cocommutative locally compact quantum group $\left(W^{*}(K), \Delta_{K}\right)$ with respect to the trivial action, i.e. it satisfies $\left(\Delta_{K} \otimes \mathrm{id}_{B\left(L^{2}(\mathcal{G}, v)\right)}\right)\left(U_{c}\right)=\left(U_{c}\right)_{23}\left(U_{c}\right)_{13}$.

Proof. From the definition of $U_{c}$, it is clear that $U_{c}$ commutes with the operators $\rho_{K}(k) \otimes 1$ for $k \in K$, where $\rho_{K}$ stands for the right regular representation of $K$. So it belongs to $W^{*}(K) \otimes B\left(L^{2}(\mathcal{G}, v)\right)$. Recall (see $\left.\S 2.2\right)$ that we have

$$
\Delta_{K}(x)=W_{K}^{*}(1 \otimes x) W_{K} \quad\left(x \in W^{*}(K)\right) .
$$

Hence, for the claimed identity, it suffices to show

$$
\left(W_{K}^{*}\right)_{12}\left(U_{c}\right)_{23}\left(W_{K}\right)_{12}=\left(U_{c}\right)_{23}\left(U_{c}\right)_{13} .
$$

However, this is straightforward.

We also fix a Borel 2-cocycle $\sigma$ on $\mathcal{G}$. With $U_{c}$ defined above, define an injective unital *-isomorphism $\alpha_{c}$ from $W^{*}(\mathcal{G}, \sigma)$ into $B\left(L^{2}(K)\right) \otimes B\left(L^{2}(\mathcal{G}, v)\right)$ by

$$
\alpha_{c}(X)=U_{c}(1 \otimes X) U_{c}^{*} \quad\left(X \in W^{*}(\mathcal{G}, \sigma)\right) .
$$

THEOREM 4.2. The morphism $\alpha_{c}$ constructed above is a coaction of $K$ on $W^{*}(\mathcal{G}, \sigma)$. 
Proof. Referring to the preceding lemma, it is enough to show that $\alpha_{c}$ maps $W^{*}(\mathcal{G}, \sigma)$ into $W^{*}(K) \otimes W^{*}(\mathcal{G}, \sigma)$.

Let $f \in \mathfrak{A}_{I}$. Then, for any $\eta \in L^{2}(K)$ and $\zeta \in \mathfrak{A}_{I}$, we have

$$
\left\{\alpha_{c}\left(L^{\sigma}(f)\right)(\eta \otimes \zeta)\right\}(k, \gamma)=\int f\left(\gamma_{1}\right) \eta\left(c\left(\gamma_{1}\right)^{-1} k\right) \zeta\left(\gamma_{1}^{-1} \gamma\right) \sigma\left(\gamma_{1}, \gamma_{1}^{-1} \gamma\right) d \lambda^{r(\gamma)}\left(\gamma_{1}\right) .
$$

This identity shows that the map $f \in \mathfrak{A}_{I} \mapsto \alpha_{c}\left(L^{\sigma}(f)\right)$ is the 'integrated form' of a groupoid representation of the tensor product of the following two representations of $\mathcal{G}$ (cf. $[15, \S 3])$ :

$$
\begin{gathered}
\lambda^{c}: \gamma \in \mathcal{G} \longmapsto \lambda_{K}(c(\gamma)) \in B\left(L^{2}(K)\right), \\
\lambda^{\sigma}(\cdot) \in B\left(L^{2}\left(\mathcal{G}^{s(\cdot)}, \lambda^{s(\cdot)}\right), L^{2}\left(\mathcal{G}^{r \cdot(\cdot)}, \lambda^{r(\cdot)}\right)\right),
\end{gathered}
$$

where $\left\{\lambda^{\sigma}\left(\gamma_{1}\right) \xi\right\}(\gamma):=\sigma\left(\gamma_{1}, \gamma_{1}^{-1} \gamma\right) \xi\left(\gamma_{1}^{-1} \gamma\right)\left(\xi \in L^{2}\left(\mathcal{G}^{s\left(\gamma_{1}\right)}, \lambda^{s\left(\gamma_{1}\right)}\right)\right)$. (Namely, $\lambda^{\sigma}$ is the $\sigma$-regular representation of $\mathcal{G}[\mathbf{1 5}])$. Thus, symbolically, we have $\alpha_{c}\left(L^{\sigma}(f)\right)=$ $\left(\lambda^{c} \otimes \lambda^{\sigma}\right)(f)$. It is then easy to see (cf. [15, Theorem 3.8, Theorem 4.1]) that the operators of the form $\left(\lambda^{c} \otimes \lambda^{\sigma}\right)(f)$ belong to $W^{*}(K) \otimes W^{*}(\mathcal{G}, \sigma)$.

Corollary 4.3. The abelian von Neumann subalgebra $L^{\infty}(X, \mu)$ is contained in the fixed-point algebra $W^{*}(\mathcal{G}, \sigma)^{\alpha_{c}}$.

One can also 'lift' the 2-cocycle $\sigma$ to the one on $\mathcal{G}_{c}$ and denote it by $\sigma_{c}$. In what follows, we will examine the groupoid von Neumann algebra $W^{*}\left(\mathcal{G}_{c}, \sigma_{c}\right)$.

We write $\mathfrak{A}_{c}$ for the $\mathcal{G}_{c}$-version of $\mathfrak{A}_{I}$ introduced before.

For any function $\phi$ on $K$ and any function $f$ on $\mathcal{G}$, define a function $\phi \times f$ on $\mathcal{G}_{c}$ by

$$
(\phi \times f)(k, \gamma):=\phi(k) f(\gamma) .
$$

It is easy to check that, if $\phi$ is in $L^{\infty}(K) \cap L^{2}(K)$ and $f$ is in $\mathfrak{A}_{I}$, the function $\phi \times f$ belongs to $\mathfrak{A}_{c}$. We denote by $\mathfrak{B}$ the self-adjoint subalgebra of $\mathfrak{A}_{c}$ generated by the functions $\phi \times f$, where $\phi \in L^{\infty}(K) \cap L^{2}(K)$ and $f \in \mathfrak{A}_{I}$.

LEMma 4.4. The subalgebra $\mathfrak{B}$ is dense in $\mathfrak{A}_{c}$ with respect to the graph norm of the S-operator.

Proof. Let $F \in \mathfrak{A}_{c}$ be such that $(\phi \times f \mid F)+\left(F^{\sharp} \mid(\phi \times f)^{\sharp}\right)=0$ for all $\phi \in L^{\infty}(K) \cap L^{2}(K)$ and all $f \in \mathfrak{A}_{I}$. For each $k \in K$, define a function $F_{k}$ on $\mathcal{G}$ by

$$
F_{k}(\gamma):=F(k, \gamma) \quad(\gamma \in \mathcal{G}) .
$$

It is easily checked that both $F_{k}$ and $F_{k}^{\sharp}$ belong to $L^{2}(\mathcal{G}, v)$ for almost every $k \in K$. First, we have

$$
\begin{aligned}
(\phi \times f \mid F) & =\int_{K} \int_{\mathcal{G}} \phi(k) f(\gamma) \overline{F(k, \gamma)} d \nu(\gamma) d k \\
& =\int_{K} \phi(k)\left(f \mid F_{k}\right) d k
\end{aligned}
$$


Second, we have

$$
\begin{aligned}
\left(F^{\sharp} \mid(\phi \times f)^{\sharp}\right) & =\int_{K} \int_{\mathcal{G}} \delta(\gamma)^{-2} \overline{F\left(c(\gamma)^{-1} k, \gamma^{-1}\right)} \phi\left(c(\gamma)^{-1} k\right) f\left(\gamma^{-1}\right) d \nu(\gamma) d k \\
& =\int_{K} \phi(k)\left(\int_{\mathcal{G}} \delta(\gamma)^{-2} \overline{F_{k}\left(\gamma^{-1}\right)} f\left(\gamma^{-1}\right) d \nu(\gamma)\right) d k \\
& =\int_{K} \phi(k)\left(\int_{\mathcal{G}} F_{k}^{\sharp}(\gamma) \overline{f^{\sharp}(\gamma)}\right) d k \\
& =\int_{K} \phi(k)\left(F_{k}^{\sharp} \mid f^{\sharp}\right) d k .
\end{aligned}
$$

Hence we obtain

$$
\int_{K} \phi(k)\left\{\left(F_{k} \mid f\right)+\left(F_{k}^{\sharp} \mid f^{\sharp}\right)\right\} d k=0
$$

for all $\phi \in L^{\infty}(K) \cap L^{2}(K)$ and all $f \in \mathfrak{A}_{I}$. Since $\mathfrak{A}_{I}$ is dense in the domain of the $S$-operator, which is separable, we may choose a sequence $\left\{f_{n}\right\}$ in $\mathfrak{A}_{I}$ which is dense in the domain of the $S$-operator. It follows from the identity above that, for almost every $k \in K$, we have $\left(F_{k} \mid f_{n}\right)+\left(F_{k}^{\sharp} \mid f_{n}^{\sharp}\right)=0$ for all $n \geq 1$. Therefore we get $F_{k}=0$ for almost every $k \in K$. Consequently, $F=0$.

COROllary 4.5. We have $\mathfrak{B}^{\prime \prime}=\mathfrak{A}_{c}^{\prime \prime}$ as left Hilbert algebras. In particular, the left von Neumann algebra of the left Hilbert algebra $\mathfrak{B}$ is $W^{*}\left(\mathcal{G}_{c}, \sigma_{c}\right)$.

Proposition 4.6. The crossed product $\widehat{K}_{\alpha_{c}} \ltimes W^{*}(\mathcal{G}, \sigma)$ is isomorphic to $W^{*}\left(\mathcal{G}_{c}, \sigma_{c}\right)$.

Proof. By Corollary $4.5, W^{*}\left(\mathcal{G}_{c}, \sigma_{c}\right)$ is generated by the operators of the form $L^{\sigma_{c}}(\phi \times f)$, where $\phi \in L^{\infty}(K) \cap L^{2}(K)$ and $f \in \mathfrak{A}_{I}$. Take another $\phi_{1} \in C_{c}(K)$ and $g \in \mathfrak{A}_{I}$. Then, by equation (4.1), we have

$$
\begin{aligned}
\left\{L^{\sigma_{c}}(\phi\right. & \left.\times f)\left(\phi_{1} \times g\right)\right\}(k, \gamma) \\
& =\int(\phi \times f)\left(k, \gamma_{1}\right)\left(\phi_{1} \times g\right)\left(c\left(\gamma_{1}\right)^{-1} k, \gamma_{1}^{-1} \gamma\right) \sigma\left(\gamma_{1}, \gamma_{1}^{-1} \gamma\right) d \lambda^{r(\gamma)}\left(\gamma_{1}\right) \\
& =\phi(k) \int f\left(\gamma_{1}\right) \phi_{1}\left(c\left(\gamma_{1}\right)^{-1} k\right) g\left(\gamma_{1}^{-1} \gamma\right) \sigma\left(\gamma_{1}, \gamma_{1}^{-1} \gamma\right) d \lambda^{r(\gamma)}\left(\gamma_{1}\right) \\
& =\left\{(\phi \otimes 1) \alpha_{c}\left(L^{\sigma}(f)\right)\left(\phi_{1} \times g\right)\right\}(k, \gamma) .
\end{aligned}
$$

Hence we obtain $L^{\sigma_{c}}(\phi \times f)=(\phi \otimes 1) \alpha_{c}\left(L^{\sigma}(f)\right)$. Thus we are done.

Recall that, under the situation as above, there is a canonical action of $K$ on the center of $W^{*}\left(\mathcal{G}_{c}, \sigma_{c}\right)$, called the Mackey action of $c[13,14]$. (This action is referred to also as the Poincaré flow of $c$ or the range of $c[8]$.)

COROLlaRY 4.7. If $\mathcal{G}$ is principal, then the Mackey action of $c$ is the restriction of the dual action $\widehat{\left(\alpha_{c}\right)}$ of $\alpha_{c}$ to the center of $\widehat{K} \alpha_{c} \ltimes W^{*}(\mathcal{G}, \sigma)$.

Now we consider another Borel 1-cocycle $c^{\prime}: \mathcal{G} \rightarrow K$ which is cohomologous to $c$. So there exists a Borel map $\phi: X \rightarrow K$ such that $c^{\prime}(\gamma)=\phi(r(\gamma)) c(\gamma) \phi(s(\gamma))^{-1}$ for almost every $\gamma \in \mathcal{G}$. By using $\phi$, we introduce a unitary $V$ on $L^{2}(K) \otimes L^{2}(\mathcal{G}, v)$ by

$$
\{V \xi\}(k, \gamma):=\xi\left(\phi(r(\gamma))^{-1} k, \gamma\right) \quad\left(\xi \in L^{2}(K \times \mathcal{G})\right) \text {. }
$$


Since $V$ can be regarded as a bounded Borel function $\gamma \in \mathcal{G} \mapsto \lambda_{K}(\phi(r(\gamma)))$, it follows that: (i) $V$ belongs to $W^{*}(K) \otimes W^{*}(\mathcal{G}, \sigma)$, (ii) it satisfies $\left(\Delta_{K} \otimes \operatorname{id}_{W^{*}(\mathcal{G}, \sigma)}\right)(V)=V_{23} V_{13}$. In the meantime, with the notation in the proof of Theorem 4.2, one has

$\left(\lambda_{K}(\phi(r(\gamma))) \otimes 1\right)\left(\lambda^{c} \otimes \lambda^{\sigma}\right)(\gamma)=\left(\lambda^{c^{\prime}} \otimes \lambda^{\sigma}\right)(\gamma)\left(\lambda_{K}(\phi(s(\gamma))) \otimes 1\right) \quad$ (almost everywhere).

This identity implies that

$$
V \alpha_{c}\left(L^{\sigma}(f)\right)=\alpha_{c^{\prime}}\left(L^{\sigma}(f)\right) V .
$$

Therefore, we obtain the following.

PROPOSITION 4.8. The action $\alpha_{c}$ is cocycle conjugate to $\alpha_{c^{\prime}}$. Hence the isomorphism class of the crossed product $\widehat{K}_{\alpha_{c}} \ltimes W^{*}(\mathcal{G}, \sigma)$ depends only on the cohomology class [c] of the Borel 1-cocycle c.

Proof. We have already shown $\operatorname{Ad} V \circ \alpha_{c}=\alpha_{c^{\prime}}$. Hence $\alpha_{c}$ is cocycle conjugate to $\alpha_{c^{\prime}}$. Since cocycle conjugate actions produce isomorphic crossed products, the second half of the assertion follows.

5. Coactions whose fixed-point algebras contain special maximal abelian $*$-subalgebras In the last section, we saw that every 1-cocycle from a measured groupoid into a locally compact group gives rise to a coaction of the group on the corresponding groupoid von Neumann algebra $A$. One of the features of the coaction so obtained was that the fixed-point algebra contains the diagonal subalgebra $D$ of $A$ (Corollary 4.3). We now ask ourselves whether any coaction of the group on $A$ whose fixed-point algebra contains $D$ arises in this way. The main purpose of this section is to prove that this is indeed the case, provided that the groupoid is principal (i.e. a measured equivalence relation).

Measured equivalence relations arise naturally from group actions on measure spaces. Suppose that a locally compact group $K$ acts on a (standard) Borel space $(X, \mathfrak{B}, \mu)$ in such a way that $G$ preserves the measure class of $\mu$. Then the graph $\mathcal{R}:=\{(k x, x) \in$ $X \times X: x \in X, k \in K\}$ of the equivalence relation induced by the action of $K$ is a typical example of a measured equivalence relation. When $K$ is a countable discrete group, every equivalence class is a countable set. Such a measured equivalence relation is said to be discrete (cf. $[\mathbf{9 , 1 0 ]}$ ). If $K$ is a continuous group whose action on $X$ is essentially free, then the measured equivalence relation $\mathcal{R}$ defined above clearly has uncountable equivalence classes. For example, the left (or right) translation action of $K$ on itself is essentially free. See also [1, Proposition 1.2]. Another typical example of a measured equivalence relation with uncountable equivalence classes is obtained as follows: let $\mathcal{R}_{0}$ be a discrete measured equivalence relation. Also consider the transitive equivalence relation $\mathbf{R}^{2}=\mathbf{R} \times \mathbf{R}$. Then define $\mathcal{R}_{1}:=\mathbf{R}^{2} \times \mathcal{R}_{0}$. It is obvious that $\mathcal{R}_{1}$ has uncountable equivalence classes. A relation between measured equivalence relations of the types $\mathcal{R}$ and $\mathcal{R}_{1}$ mentioned above is thoroughly discussed in [8] (see also [24]).

Let us fix a coaction $\alpha$ of a locally compact group $K$ on a von Neumann algebra $A$. We will assume in what follows that $A$ is expressed in the form $W^{*}(\mathcal{R}, \sigma)$, where $\mathcal{R}$ is a measured equivalence relation over a standard Borel measure space $(X, \mu)$, 
and $\sigma$ is a Borel 2-cocycle on $\mathcal{R}$. We also make an assumption that $A^{\alpha}$ contains the diagonal subalgebra $D:=L^{\infty}(X, \mu)$.

We also retain the notation introduced in the preceding section.

As we mentioned above, our goal in this section is to find a 1-cocycle $c: \mathcal{R} \rightarrow K$ satisfying $\alpha=\alpha_{c}$ in the notation in $\S 4$. However, before we proceed, let us remark that if the groupoid $\mathcal{R}$ is the equivalence relation generated by an essentially free action of a countable discrete group $\Gamma$ on $X$, then such a 1-cocycle $c: \mathcal{R} \rightarrow K$ is relatively easily obtained. In fact, if $\{u(\gamma)\}_{\gamma \in \Gamma}$ are the unitaries in $A=\Gamma \ltimes L^{\infty}(X)$ implementing the $\Gamma$-action on $L^{\infty}(X, \mu)$, then the same argument as in the proof of Proposition 3.1 shows that $\left(1 \otimes u(\gamma)^{*}\right) \alpha(u(\gamma))$ belongs to $W^{*}(K) \otimes L^{\infty}(X)=L^{\infty}\left(X, W^{*}(K)\right)$, so that there is a Borel map $c: \Gamma \times X \rightarrow K$ such that

$$
\left(1 \otimes u(\gamma)^{*}\right) \alpha(u(\gamma))=\lambda_{K}(c(\gamma, \cdot)) \quad(\gamma \in \Gamma) .
$$

It is easy to check that $c$ is a 1-cocycle on the groupoid $\Gamma \times X \cong \mathcal{R}$, and that $\alpha=\alpha_{c}$. It is, however, not true in general that a principal measured groupoid is realized as the graph of an equivalence relation induced by an essentially free group action on a measure space (see [12]). Hence it seems that a lot more work is needed to achieve our goal.

Let $\varphi_{\mathcal{R}}$ denote the faithful normal semifinite weight on $W^{*}(\mathcal{R}, \sigma)$ associated to the Tomita algebra $\mathfrak{A}_{I}$. We call this weight the Plancherel weight of $\mathcal{R}$. Refer to [35] for basic facts about Plancherel weights. The modular operator and modular conjugation of $\varphi_{\mathcal{R}}$ are given in the previous section. Let $U$ be the canonical implementation of the coaction $\alpha$ associated to $\varphi_{\mathcal{R}}$ in the sense of [33]. So we have $U=\tilde{J}\left(J_{K} \otimes J\right)$, where $\tilde{J}$ is the modular conjugation of the dual weight $\widetilde{\varphi_{\mathcal{R}}}$ defined on the crossed product $\widehat{K}_{\alpha} \ltimes W^{*}(\mathcal{R}, \sigma)$ and $J_{K}$ is defined on $L^{2}(K)$ by $\left\{J_{K} f\right\}(k)=\overline{f(k)}$. Then $U$ induces a $*$-representation $\Pi$ of the Fourier algebra $A(K)=W^{*}(K)_{*}$ of $K$ (see [7]) on $L^{2}(\mathcal{R}, v)$ given by

$$
\Pi(\omega):=\left(\omega \otimes \operatorname{id}_{B\left(L^{2}(\mathcal{R}, v)\right)}\right)\left(U^{*}\right) \quad(\omega \in A(K)) .
$$

We know from [7] that the spectrum of the abelian Banach $*$-algebra $A(K)$ is $K$. Hence, by [11, Theorem 1.54], there is a unique $L^{2}(\mathcal{R}, v)$-projection-valued measure $P$ on $K$ such that

$$
\Pi(\omega)=\int_{K} \hat{\omega} d P\left(=\int_{K} \omega\left(\lambda_{K}(k)\right) d P(k)\right) .
$$

LEMMA 5.1. The projection-valued measure $P$ is induced by a Borel measurable map $\phi: \mathcal{R} \longrightarrow K$ in such a way that $P(B)=\chi_{\phi^{-1}\left(B^{-1}\right)}$ for all Borel subsets $B$ of $K$.

Proof. Since $D$ is contained in $A^{\alpha}, U$ commutes with all the operators of the form $1 \otimes a$, where $a \in D$. It follows from this and the definition of $\Pi$ that $D$ is contained in $\Pi(A(K))^{\prime}$. Since $U^{*}=\left(J_{K} \otimes J\right) U\left(J_{K} \otimes J\right), U$ commutes also with the operators of the form $1 \otimes J a J$, where $a \in D$. Hence $J D J$ is also contained in $\Pi(A(K))^{\prime}$. By [15], we have $D \vee J D J=L^{\infty}(\mathcal{R}, v)$. This shows that $\Pi(A(K))^{\prime \prime}$ is contained in $L^{\infty}(\mathcal{R}, v)$. In particular, $P(B)$ is a projection in $L^{\infty}(\mathcal{R}, v)$ for all Borel subsets $B$ of $K$. Therefore $E \mapsto P(E)$ is an assignment from the Borel $\sigma$-algebra of $K$ into the lattice of all the projections in $L^{\infty}(\mathcal{R}, v)$. By [26, Ch. 15, Proposition 19], there is a Borel measurable map $\phi$ from $\mathcal{R}$ into $K$ such that $P(B)=\chi_{\phi^{-1}\left(B^{-1}\right)}$ for all Borel subsets $B$ of $K$. 
COROLlary 5.2. The canonical implementation $U$ of $\alpha$ belongs to $W^{*}(K) \otimes L^{\infty}(\mathcal{R}, v)$, and it can be regarded as a bounded Borel function $\gamma \in \mathcal{R} \longmapsto \lambda_{K}(\phi(\gamma)) \in W^{*}(K)$. Moreover, we have

$$
\begin{aligned}
& \left\{\alpha\left(L^{\sigma}(f)\right)(\eta \otimes \zeta)\right\}(k, \gamma) \\
& \quad=\int \eta\left(\phi\left(\gamma_{1}^{-1} \gamma\right) \phi(\gamma)^{-1} k\right) f\left(\gamma_{1}\right) \zeta\left(\gamma_{1}^{-1} \gamma\right) \sigma\left(\gamma_{1}, \gamma_{1}^{-1} \gamma\right) d \lambda^{r(\gamma)}\left(\gamma_{1}\right)
\end{aligned}
$$

for all $\eta \in L^{2}(K)$ and all $f, \zeta \in \mathfrak{A}_{I}$.

Proof. By (the proof of) Lemma 5.1, we see that $U^{*}$ belongs to $W^{*}(K) \otimes L^{\infty}(\mathcal{R})$, and that it can be regarded as a bounded Borel function $\gamma \in \mathcal{R} \longmapsto \lambda_{K}(\phi(\gamma))^{*} \in W^{*}(K)$. Equation (5.1) follows from a direct computation by using the fact that $\alpha(T)=$ $U(1 \otimes T) U^{*}$.

LEMMA 5.3. The Plancherel weight $\varphi_{\mathcal{R}}$ is 1-invariant with respect to $\alpha$ in the sense of [33, Definition 2.3]. Namely, for any $T \in \mathfrak{m}_{\varphi_{\mathcal{R}}}^{+}$and $\eta \in L^{2}(K)$, we have

$$
\varphi_{\mathcal{R}}\left(\left(\omega_{\eta} \otimes \operatorname{id}_{A}\right)(\alpha(T))\right)=\|\eta\|^{2} \varphi_{\mathcal{R}}(T)
$$

Proof. Define $\mathcal{P}$ to be the set of all right-bounded vectors $\zeta \in L^{2}(\mathcal{R}, v)$ with respect to the Tomita algebra $\mathfrak{A}_{I}$ such that $\left\|R^{\sigma}(\zeta)\right\| \leq 1$. Then we have

$$
\varphi_{\mathcal{R}}(T)=\sup \left\{\omega_{\zeta}(T): \zeta \in \mathcal{P}\right\}
$$

for any $T \in W^{*}(\mathcal{R}, \sigma)_{+}$.

Let $f \in \mathfrak{A}_{I}, \eta \in L^{2}(K)$ and $\zeta \in \mathcal{P}$. Then, by Corollary 5.2, we have

$$
\begin{aligned}
\omega_{\zeta}\left(\left(\omega_{\eta} \otimes \operatorname{id}_{A}\right)\left(\alpha\left(L^{\sigma}(f)^{*} L^{\sigma}(f)\right)\right)\right) \\
\quad=\left\|\alpha\left(L^{\sigma}(f)\right)(\eta \otimes \zeta)\right\|^{2} \\
\quad=\iint\left|\int \eta\left(\phi\left(\gamma_{1}^{-1} \gamma\right) \phi(\gamma) k\right)^{-1} f\left(\gamma_{1}\right) \zeta\left(\gamma_{1}^{-1} \gamma\right) \sigma\left(\gamma_{1}, \gamma_{1}^{-1} \gamma\right) d \lambda^{r(\gamma)}\left(\gamma_{1}\right)\right|^{2} d k d \nu(\gamma) \\
\quad=\iint\left|\int \eta(k) f\left(\gamma_{1}\right) \zeta\left(\gamma_{1}^{-1} \gamma\right) \sigma\left(\gamma_{1}, \gamma_{1}^{-1} \gamma\right) d \lambda^{r(\gamma)}\left(\gamma_{1}\right)\right|^{2} d k d \nu(\gamma) \\
\quad=\|\eta\|^{2} \int\left|\int f\left(\gamma_{1}\right) \zeta\left(\gamma_{1}^{-1} \gamma\right) \sigma\left(\gamma_{1}, \gamma_{1}^{-1} \gamma\right) d \lambda^{r(\gamma)}\left(\gamma_{1}\right)\right|^{2} d \nu(\gamma) \\
\quad=\|\eta\|^{2}\|f * \zeta\|^{2}=\|\eta\|^{2}\left\|R^{\sigma}(\zeta) f\right\|^{2}
\end{aligned}
$$

Let $f_{0} \in L^{2}(\mathcal{R}, v)$ be a left bounded vector. Then, there exists a sequence $\left\{f_{n}\right\}_{n \geq 1}$ in $\mathfrak{A}_{I}$ such that $\lim _{n \rightarrow \infty}\left\|f_{0}-f_{n}\right\|=0$ and strong- $\lim _{n \rightarrow \infty} L^{\sigma}\left(f_{n}\right)=L^{\sigma}\left(f_{0}\right)$. By the above computation, we obtain

$$
\begin{aligned}
\omega_{\zeta}\left(\left(\omega_{\eta} \otimes \operatorname{id}_{A}\right)\left(\alpha\left(L^{\sigma}\left(f_{0}\right)^{*} L^{\sigma}\left(f_{0}\right)\right)\right)\right) & =\left\|\alpha\left(L^{\sigma}\left(f_{0}\right)\right)(\eta \otimes \zeta)\right\|^{2} \\
& =\lim _{n \rightarrow \infty}\left\|\alpha\left(L^{\sigma}\left(f_{n}\right)\right)(\eta \otimes \zeta)\right\|^{2} \\
& =\|\eta\|^{2} \lim _{n \rightarrow \infty}\left\|R^{\sigma}(\zeta) f_{n}\right\|^{2}=\|\eta\|^{2}\left\|R^{\sigma}(\zeta) f_{0}\right\|^{2}
\end{aligned}
$$


From this, it follows that

$$
\begin{aligned}
\varphi_{\mathcal{R}}\left(\left(\omega_{\eta} \otimes \operatorname{id}_{A}\right)\left(\alpha\left(L^{\sigma}\left(f_{0}\right)^{*} L^{\sigma}\left(f_{0}\right)\right)\right)\right) \\
\quad=\sup \left\{\omega_{\zeta}\left(\left(\omega_{\eta} \otimes \operatorname{id}_{A}\right)\left(\alpha\left(L^{\sigma}\left(f_{0}\right)^{*} L^{\sigma}\left(f_{0}\right)\right)\right)\right): \zeta \in \mathcal{P}\right\} \\
\quad=\sup \left\{\|\eta\|^{2}\left\|R^{\sigma}(\zeta) f_{0}\right\|^{2}: \zeta \in \mathcal{P}\right\} \\
\quad=\|\eta\|^{2}\left\|f_{0}\right\|^{2}=\|\eta\|^{2} \varphi_{\mathcal{R}}\left(L^{\sigma}\left(f_{0}\right)^{*} L^{\sigma}\left(f_{0}\right)\right)
\end{aligned}
$$

This proves the lemma.

COROllaRY 5.4. Let $\tilde{\nabla}$ be the modular operator of the dual weight of $\varphi_{\mathcal{R}}$, defined on $\widehat{K}_{\alpha} \ltimes W^{*}(\mathcal{R}, \sigma)$. Then we have $\tilde{\nabla}=1 \otimes \nabla$.

Proof. We know from Lemma 5.3 that $\varphi_{\mathcal{R}}$ is 1 -invariant. So, as in the proof of [33, Proposition 4.3], we can show that $\tilde{\nabla}=1 \otimes \nabla$. (Note that, in our case, the operator $Q$ in the proof of [33, Proposition 4.3] is 1.)

LEMMA 5.5. The modular conjugation $\tilde{J}$ of $\widetilde{\varphi_{\mathcal{R}}}$ is given by

$$
\{\tilde{J} \xi\}(k, \gamma)=\sigma\left(\gamma, \gamma^{-1}\right)^{-1} \delta(\gamma)^{-1 / 2} \overline{\xi\left(\phi(\gamma)^{-1} k, \gamma^{-1}\right)}
$$

for any $\xi \in L^{2}(K) \otimes L^{2}(\mathcal{R}, v)=L^{2}(K \times \mathcal{R})$.

Proof. The claim follows from the fact that $\tilde{J}=U\left(J_{K} \otimes J\right)$.

COROLlaRY 5.6. We have $\phi(\gamma)^{-1}=\phi\left(\gamma^{-1}\right)$ for almost every $\gamma \in \mathcal{R}$.

Proof. This follows from the previous lemma and the fact that $\tilde{J}^{2}=1$.

LEMMA 5.7. The map $\phi$ satisfies

$$
\phi\left(\gamma_{1} \gamma_{2}\right)=\phi\left(\gamma_{1}\right) \phi\left(\gamma_{2}\right)
$$

for $v^{(2)}$-almost every $\left(\gamma_{1}, \gamma_{2}\right) \in \mathcal{R}^{(2)}$.

Proof. Let $f \in \mathfrak{A}_{I}, p \in C_{c}(K)$. We first regard $W^{*}(K) \otimes W^{*}(\mathcal{R}, \sigma)$ as being equal to the groupoid von Neumann algebra $W^{*}(K \times \mathcal{R}, 1 \times \sigma)$, where $K \times \mathcal{R}$ is equipped with the natural product measured groupoid structure. By Lemma 5.3 and [33, Proposition 2.4], we have that $\alpha\left(L^{\sigma}(f)\right)(L(p) \otimes 1)$ belongs to $\mathfrak{n}_{\varphi_{K} \otimes \varphi_{\mathcal{R}}}$ and satisfies

$$
\Lambda_{\varphi_{K} \otimes \varphi_{\mathcal{R}}}\left(\alpha\left(L^{\sigma}(f)\right)(L(p) \otimes 1)\right)=U \Lambda_{\varphi_{K} \otimes \varphi_{\mathcal{R}}}\left(L(p) \otimes L^{\sigma}(f)\right)(=U(p \otimes f)) .
$$

Thus $\alpha\left(L^{\sigma}(f)\right)(L(p) \otimes 1)$ is equal to $L^{1 \times \sigma}(F)$, where $F \in L^{2}(K \times \mathcal{R})$ is the leftbounded vector $U(p \otimes f)$ with respect to the left Hilbert algebra $C_{c}(K) \otimes \mathfrak{A}_{I}$. So we have $F(k, \gamma)=p\left(\phi(\gamma)^{-1} k\right) f(\gamma)$. In particular, by Fubini's theorem and Corollary 5.6, 
for any $\eta \in C_{c}(K)$ and $\zeta \in \mathfrak{A}_{I}$, we have the following:

$$
\begin{aligned}
\alpha\left(L^{\sigma}(f)\right)(L(p) \otimes 1)(\eta \otimes \zeta)(k, \gamma) \\
=\{F *(\eta \otimes \zeta)\}(k, \gamma) \\
=\iint F\left(h, \gamma_{1}\right)(\eta \otimes \zeta)\left(h^{-1} k, \gamma_{1}^{-1} \gamma\right) \sigma\left(\gamma_{1}, \gamma_{1}^{-1} \gamma\right) d h d \lambda^{r(\gamma)}\left(\gamma_{1}\right) \\
=\iint f\left(\gamma_{1}\right) p\left(\phi\left(\gamma_{1}\right)^{-1} h\right) \eta\left(h^{-1} k\right) \zeta\left(\gamma_{1}^{-1} \gamma\right) \sigma\left(\gamma_{1}, \gamma_{1}^{-1} \gamma\right) d h d \lambda^{r(\gamma)}\left(\gamma_{1}\right) \\
=\iint f\left(\gamma_{1}\right) p(h) \eta\left(h^{-1} \phi\left(\gamma_{1}\right)^{-1} k\right) \zeta\left(\gamma_{1}^{-1} \gamma\right) \sigma\left(\gamma_{1}, \gamma_{1}^{-1} \gamma\right) d h d \lambda^{r(\gamma)}\left(\gamma_{1}\right) \\
=\int(p * \eta)\left(\phi\left(\gamma_{1}\right)^{-1} k\right) f\left(\gamma_{1}\right) \zeta\left(\gamma_{1}^{-1} \gamma\right) \sigma\left(\gamma_{1}, \gamma_{1}^{-1} \gamma\right) d \lambda^{r(\gamma)}\left(\gamma_{1}\right) \\
=\int(p * \eta)\left(\phi\left(\gamma_{1}^{-1}\right) k\right) f\left(\gamma_{1}\right) \zeta\left(\gamma_{1}^{-1} \gamma\right) \sigma\left(\gamma_{1}, \gamma_{1}^{-1} \gamma\right) d \lambda^{r(\gamma)}\left(\gamma_{1}\right) .
\end{aligned}
$$

On the other hand, by using Corollary 5.2, we have that

$$
\begin{aligned}
& \alpha\left(L^{\sigma}(f)\right)(L(p) \otimes 1)(\eta \otimes \zeta)(k, \gamma) \\
& \quad=\alpha\left(L^{\sigma}(f)\right)((p * \eta) \otimes \zeta)(k, \gamma) \\
& \quad=\int(p * \eta)\left(\phi\left(\gamma_{1}^{-1} \gamma\right) \phi(\gamma)^{-1} k\right) f\left(\gamma_{1}\right) \zeta\left(\gamma_{1}^{-1} \gamma\right) \sigma\left(\gamma_{1}, \gamma_{1}^{-1} \gamma\right) d \lambda^{r(\gamma)}\left(\gamma_{1}\right) .
\end{aligned}
$$

It follows that, for almost every $(k, \gamma) \in K \times \mathcal{R}$, the following equation holds:

$$
\begin{aligned}
& \int f\left(\gamma_{1}\right) \zeta\left(\gamma_{1}^{-1} \gamma\right)\left((p * \eta)\left(\phi\left(\gamma_{1}^{-1}\right) k\right)\right. \\
& \left.\quad-(p * \eta)\left(\phi\left(\gamma_{1}^{-1} \gamma\right) \phi(\gamma)^{-1} k\right)\right) \sigma\left(\gamma_{1}, \gamma_{1}^{-1} \gamma\right) d \lambda^{r(\gamma)}\left(\gamma_{1}\right)=0 .
\end{aligned}
$$

From these results and the facts that $C_{c}(K) * C_{c}(K)$ is dense in $C_{c}(K)$ and the Haar measure is left invariant, we conclude that, for each $\eta, \eta_{1} \in C_{c}(K)$ and $f, g \in \mathfrak{A}_{I}$, we have

$$
\int\left(F_{\eta}\left(\gamma, \gamma_{1}, \cdot\right) \mid \eta_{1}\right)_{L^{2}(K)} f\left(\gamma_{1}\right) g\left(\gamma_{1}^{-1} \gamma\right) \sigma\left(\gamma_{1}, \gamma_{1}^{-1} \gamma\right) d \lambda^{r(\gamma)}\left(\gamma_{1}\right)=0
$$

for almost every $\gamma \in \mathcal{R}$, where $F_{\eta}$ is the function on $\mathcal{R} \times \mathcal{R} \times K$ given by

$$
F_{\eta}\left(\gamma, \gamma_{1}, k\right):=\eta\left(\phi\left(\gamma_{1}^{-1}\right) \phi(\gamma) k\right)-\eta\left(\phi\left(\gamma_{1}^{-1} \gamma\right) k\right) \text {. }
$$

Let $\left\{f_{n, m}\right\}_{n, m \geq 1}$ be the sequence of Borel functions in $\mathfrak{A}_{I}$ defined in the proof of [34, Lemma 1.5] (see also the proof of [15, Lemma 2.9]). Also choose a countable generating (and separating) Borel subsets $\left\{B_{n}\right\}_{n \geq 1}$ of $K$ of finite measure. Then there exists a null Borel subset $N_{1}$ of $\mathcal{R}$ such that

$$
\int\left(F_{\chi_{B_{i}}}\left(\gamma, \gamma_{1}, \cdot\right) \mid \chi_{B_{j}}\right) f_{k, l}\left(\gamma_{1}\right) f_{m, n}^{\vee}\left(\gamma_{1}^{-1} \gamma\right) \sigma\left(\gamma_{1}, \gamma_{1}^{-1} \gamma\right) d \lambda^{r(\gamma)}\left(\gamma_{1}\right)=0
$$

for all $\gamma \in \mathcal{R} \backslash N_{1}$ and all $(i, j, k, l, m, n) \in \mathbf{N}^{6}$, where $f^{\vee}(\gamma):=f\left(\gamma^{-1}\right)$. By arguing as in the proof of [34, Lemma 1.5] or [15, Lemma 2.9], we deduce that, for any $\gamma \in \mathcal{R} \backslash N_{1}$, the function

$$
\gamma_{1} \longmapsto\left(F_{\chi_{B_{i}}}\left(\gamma, \gamma_{1}, \cdot\right) \mid \chi_{B_{j}}\right) f_{m, n}^{\vee}\left(\gamma_{1}^{-1} \gamma\right)
$$


is 0 for $\lambda^{r(\gamma)}$-almost everywhere. From this, we have

$$
\begin{aligned}
0 & =\iint\left|\left(F_{\chi_{B_{i}}}\left(\gamma, \gamma_{1}, \cdot\right) \mid \chi_{B_{j}}\right) f_{m, n}^{\vee}\left(\gamma_{1}^{-1} \gamma\right)\right| d \lambda^{r(\gamma)}\left(\gamma_{1}\right) d \nu^{-1}(\gamma) \\
& =\iint\left|\left(F_{\chi_{B_{i}}}\left(\gamma^{-1}, \gamma_{1}, \cdot\right) \mid \chi_{B_{j}}\right) f_{m, n}^{\vee}\left(\gamma_{1}^{-1} \gamma^{-1}\right)\right| d \lambda^{s(\gamma)}\left(\gamma_{1}\right) d \nu(\gamma) \\
& =\iint\left|\left(F_{\chi_{B_{i}}}\left(\gamma^{-1}, \gamma^{-1} \gamma_{1}, \cdot\right) \mid \chi_{B_{j}}\right) f_{m, n}\left(\gamma_{1}\right)\right| d \lambda^{r(\gamma)}\left(\gamma_{1}\right) d \nu(\gamma) \\
& =\int\left(\int\left|\left(F_{\chi_{B_{i}}}\left(\gamma^{-1}, \gamma^{-1} \gamma_{1}, \cdot\right) \mid \chi_{B_{j}}\right)\right| d \lambda^{r\left(\gamma_{1}\right)}(\gamma)\right) f_{m, n}\left(\gamma_{1}\right) d \nu\left(\gamma_{1}\right)
\end{aligned}
$$

(by Fubini's theorem).

It follows that there exists a $\nu$-null Borel subset $N_{2}$ of $\mathcal{R}$ with $N_{2}^{-1}=N_{2}$ such that we have

$$
\int\left|\left(F_{\chi_{B_{i}}}\left(\gamma^{-1}, \gamma^{-1} \gamma_{1}, \cdot\right) \mid \chi_{B_{j}}\right)\right| d \lambda^{r\left(\gamma_{1}\right)}(\gamma)=0
$$

for all $\gamma_{1} \in \mathcal{R} \backslash N_{2}$ and $(i, j) \in \mathbf{N}^{2}$. Fix an arbitrary $\gamma_{1} \in \mathcal{R} \backslash N_{2}$. Then there exists a $\lambda^{r\left(\gamma_{1}\right)}$-null Borel subset $N\left(\gamma_{1}\right)$ such that, for any $\gamma \in \mathcal{R}^{r\left(\gamma_{1}\right)} \backslash N\left(\gamma_{1}\right)$ and any $(i, j) \in \mathbf{N}^{2}$, one has

$$
\left(F_{\chi_{B_{i}}}\left(\gamma^{-1}, \gamma^{-1} \gamma_{1}, \cdot\right) \mid \chi_{B_{j}}\right)=0
$$

Now fix a $\gamma \in \mathcal{R}^{r\left(\gamma_{1}\right)} \backslash N\left(\gamma_{1}\right)$. By the above identity, we obtain

$$
\phi\left(\gamma_{1}^{-1}\right) \phi(\gamma)=\phi\left(\gamma_{1}^{-1} \gamma\right)
$$

Since $N_{2}=N_{2}^{-1}$, we have

$$
\phi\left(\gamma_{1} \gamma\right)=\phi\left(\gamma_{1}\right) \phi(\gamma)
$$

for all $\gamma_{1} \in \mathcal{R} \backslash N_{2}$ and all $\gamma \in \mathcal{R}^{s\left(\gamma_{1}\right)} \backslash N^{\prime}\left(\gamma_{1}\right)$, where $N^{\prime}(\gamma):=N\left(\gamma^{-1}\right)$. For any $j \in \mathbf{N}$, define a Borel function $f_{j}$ on $\mathcal{R}^{(2)}$ by

$$
f_{j}\left(\gamma_{1}, \gamma_{2}\right):=\left|\chi_{B_{j}}\left(\phi\left(\gamma_{1} \gamma_{2}\right)\right)-\chi_{B_{j}}\left(\phi\left(\gamma_{1}\right) \phi\left(\gamma_{2}\right)\right)\right| \text {. }
$$

From the result obtained above, we get

$$
\int f_{j}\left(\gamma_{1}, \gamma_{2}\right) d v^{(2)}\left(\gamma_{1}, \gamma_{2}\right)=\int\left(\int f_{j}\left(\gamma_{1}, \gamma\right) d \lambda^{s\left(\gamma_{1}\right)}(\gamma)\right) d \nu^{-1}\left(\gamma_{1}\right)=0
$$

for all $j \in \mathbf{N}$. This implies that $f_{j}=0$ for $\nu^{(2)}$-almost everywhere for all $j \in \mathbf{N}$. Therefore identity (5.2) holds for $v^{(2)}$-almost every $\left(\gamma_{1}, \gamma\right) \in \mathcal{R}^{(2)}$.

THEOREM 5.8. Let $\alpha$ be a coaction of a locally compact group $K$ on the groupoid von Neumann algebra A of a principal measured groupoid $\mathcal{R}$. Suppose that $A^{\alpha}$ contains the diagonal subalgebra $D$ of $A$. Then there exists a Borel 1-cocycle $c: \mathcal{R} \rightarrow K$ such that, with the notation in the previous section, we have $\alpha=\alpha_{c}$. In particular, any coaction of a locally compact group on a von Neumann algebra $M$ whose fixed-point algebra contains a regular maximal von Neumann subalgebra $C$ of $M$ that contains a quasi-Cartan subalgebra $B$ of $M$ is derived from a Borel 1-cocycle of the principal measured groupoid determined by the triple ( $M \supseteq C \supseteq B)$ through the result of $[\mathbf{8}]$. 
Proof. Let $\phi$ be the map obtained in Lemma 5.1. From Lemma 5.7 and [24, Theorem 3.2], there is a Borel 1-cocycle $c: \mathcal{R} \rightarrow K$ that agrees with the map $\gamma \in \mathcal{R} \mapsto \phi(\gamma) \nu$-almost everywhere. In this case, it is clear by Corollary 5.2 that the canonical implementation $U$ equals $U_{c}$. Therefore we have $\alpha=\alpha_{c}$.

The last part of the theorem follows from the first part and the remark at the end of $\S 2.1$.

COROLlary 5.9. Let the notation be as in Theorem 5.8. For each automorphism $\theta$ of $A$ such that $A^{\theta}$ contains $D$, there exists a 1-cocycle $c: \mathcal{R} \rightarrow \mathbf{T}$ such that $\theta=\theta_{c}: L^{\sigma}(f) \mapsto$ $L^{\sigma}(f c)$.

Let us continue to consider the situation set out at the beginning of this section. Suppose that we have another coaction $\alpha^{\prime}$ of $K$ on $A$ with $A^{\alpha^{\prime}} \supseteq D$. Referring to Theorem 5.8, there exists a Borel 1-cocycle $c^{\prime}: \mathcal{R} \longrightarrow K$ satisfying $\alpha^{\prime}=\alpha_{c^{\prime}}$. In the previous section, we saw that $\alpha_{c^{\prime}}$ is cocycle conjugate to $\alpha_{c}$ if $c^{\prime}$ is cohomologous to $c$. We now assume instead that $\alpha_{c^{\prime}}$ is cocycle conjugate to $\alpha_{c}$ with a special $*$-automorphism. Namely, let us suppose that there exist an $\alpha_{c}$-1-cocycle $V$ in $W^{*}(K) \otimes A$ and a $*$-automorphism $\theta$ of $A$ such that $\theta(D)=D$ and $\left(\operatorname{id}_{W^{*}(K)} \otimes \theta\right) \circ \alpha_{c^{\prime}} \circ \theta^{-1}=\operatorname{Ad} V \circ \alpha_{c}$. We first show that there exists a Borel and groupoid automorphism $\rho$ of $\mathcal{R}$ (which of course preserves the measure class $[v])$ such that $\left(\operatorname{id}_{W^{*}(K)} \otimes \theta\right) \circ \alpha_{c^{\prime}} \circ \theta^{-1}$ is equal to $\alpha_{c^{\prime} \circ \rho^{-1}}$. Indeed, let $u$ be the canonical implementation of $\theta$ on $L^{2}(\mathcal{R})$. Note then that $\operatorname{Ad} u$ leaves $D$ and $D \vee J D J=L^{\infty}(\mathcal{R}, v)$ globally invariant, where $J$ is the modular conjugation of the Plancherel weight $\varphi_{\mathcal{R}}$. Hence, by the same argument as in the proof of [10, Theorem 2], we obtain a Borel and groupoid automorphism $\rho$ of $\mathcal{R}$ which preserves the class $[v]$ and satisfies $\operatorname{Ad} u(f)=f \circ \rho^{-1}$ for all $f \in L^{\infty}(\mathcal{R}, v)$. Now we define a unitary $v$ in $B\left(L^{2}(\mathcal{R})\right)$ by

$$
(v \xi)(\gamma):=\xi\left(\rho^{-1}(\gamma)\right)\left[\frac{d v \circ \rho^{-1}}{d v}(\gamma)\right]^{1 / 2} .
$$

Since $u v^{*}$ is in $L^{\infty}(\mathcal{R})^{\prime} \cap B\left(L^{2}(\mathcal{R})\right)=L^{\infty}(\mathcal{R})$, there exists $g \in L^{\infty}(\mathcal{R})$ such that $|g|=1$ and $u=g v$. A direct calculation shows that

$$
\begin{aligned}
\left\{(1 \otimes u) U_{c^{\prime}}\left(1 \otimes u^{*}\right) \xi\right\}(k, \gamma) & =\left\{(1 \otimes g v) U_{c^{\prime}}\left(1 \otimes v^{*} \bar{g}\right) \xi\right\}(k, \gamma) \\
& =\xi\left(c^{\prime}\left(\rho^{-1}(\gamma)\right) k, \gamma\right) \\
& =\left\{U_{c^{\prime} \circ \rho^{-1}} \xi\right\}(k, \gamma)
\end{aligned}
$$

for each $\xi \in L^{2}(K \times \mathcal{R})$. So we conclude that $(1 \otimes u) U_{c^{\prime}}\left(1 \otimes u^{*}\right)=U_{c^{\prime} \circ \rho^{-1}}$ and

$$
\left(\operatorname{id}_{W^{*}(K)} \otimes \theta\right) \circ \alpha_{c^{\prime}} \circ \theta^{-1}(a)=U_{c^{\prime} \circ \rho^{-1}}(1 \otimes a) U_{c^{\prime} \circ \rho^{-1}}^{*}=\alpha_{c^{\prime} \circ \rho^{-1}}(a) \quad(a \in A) .
$$

Thus our claim has been proven. Furthermore, since both $A^{\alpha_{c}}$ and $A^{\alpha^{\prime} \circ \rho^{-1}}$ contain $D$, $V$ belongs to $W^{*}(K) \otimes A \cap(\mathbf{C} \otimes D)^{\prime}=W^{*}(K) \otimes D$. Hence the cocycle identity $\left(\Delta_{K} \otimes \operatorname{id}_{A}\right)(V)=V_{23}\left(\operatorname{id}_{W^{*}(K)} \otimes \alpha_{C}\right)(V)$ is nothing but $\left(\Delta_{K} \otimes \operatorname{id}_{A}\right)(V)=V_{23} V_{13}$. Then, by arguing as in the proof of Proposition 3.1 or as in this section, we may conclude that $V$ induces a Borel map $\phi: X \longrightarrow K$ such that $\left\{V^{*} \xi\right\}(k, \gamma)=\xi(\phi(r(\gamma)) k, \gamma)$ for any $\xi \in L^{2}(K \times \mathcal{R})$. Since $U_{c}, U_{c^{\prime} \circ \rho^{-1}}$ and $V$ satisfy the identity in [33, Proposition 4.2], it follows that we have $c^{\prime}\left(\rho^{-1}(\gamma)\right)=\phi(r(\gamma)) c(\gamma) \phi(s(\gamma))^{-1}$ for almost every $\gamma \in \mathcal{R}$. Therefore $c^{\prime}$ is weakly equivalent to $c$. Thus we have proven the following. 
PROPOSITION 5.10. Let A be the groupoid von Neumann algebra of a principal measured groupoid $\mathcal{R}$, twisted by a Borel 2-cocycle $\sigma$ on $\mathcal{R}$, and $D$ the diagonal subalgebra of $A$. Suppose that $\alpha$ and $\beta$ are coactions of a locally compact group $K$ on $A$ such that $D \subseteq A^{\alpha}$ and $D \subseteq A^{\beta}$. Let $c_{\alpha}$ (respectively $c_{\beta}$ ) be a Borel 1 -cocycle into $K$ corresponding to $\alpha$ (respectively $\beta$ ). If there exist an $\alpha$-1-cocycle $V$ and $a *$-automorphism $\theta$ of $A$ such that $\theta(D)=D$ and $\left(\operatorname{id}_{W^{*}(K)} \otimes \theta\right) \circ \beta \circ \theta^{-1}=\operatorname{Ad} V \circ \alpha$, then $c_{\alpha}$ is weakly equivalent to $c_{\beta}$.

Remark. The converse of Proposition 5.10 holds if there exists a measure-class preserving Borel and groupoid automorphism $\rho$ of $\mathcal{R}$ such that: (i) $c_{\beta} \circ \rho^{-1}$ is cohomologous to $c_{\alpha}$, (ii) $\rho$ fixes the 2-cocycle $\sigma$, (iii) the Haar system $\left\{\lambda^{x}\right\}$ of $\mathcal{R}$ satisfies $\lambda^{r(\gamma)}=\lambda^{r\left(\rho^{-1}(\gamma)\right)} \circ \rho^{-1}$ for almost every $\gamma \in \mathcal{R}$. (Note that the condition (iii) is automatically fulfilled if $\mathcal{R}$ is a discrete equivalence relation.) Indeed, if such a $\rho$ exists, then define a unitary $v$ by

$$
(v \xi)(\gamma):=\xi\left(\rho^{-1}(\gamma)\right)\left[\frac{d v \circ \rho^{-1}}{d v}(\gamma)\right]^{1 / 2} .
$$

One shows that $\theta:=\left.\operatorname{Ad} v\right|_{A}$ yields an automorphism of $A$. It is then easy to check that $\alpha_{c_{\beta} \circ \rho^{-1}}=\left(\operatorname{id}_{W^{*}(K)} \otimes \theta\right) \circ \beta \circ \theta^{-1}$.

At the beginning of [8, Section 8], Feldman et al claim without proof that any measureclass preserving Borel and groupoid automorphism $\rho$ of $\mathcal{R}$ satisfying the condition (ii) gives rise to a (spatial) automorphism $\theta_{\rho}$ of $A$ with $\theta_{\rho}(D)=D$. We have not been able to verify this claim. However, with the help of their claim, we could eliminate the condition (iii) in the above 'converse' statement.

\section{Connes spectrum and asymptotic range}

The main results of the preceding two sections together illustrate that there is an intimate relationship between Borel 1-cocycles on a measured groupoid and coactions on the groupoid von Neumann algebra that fix the diagonal subalgebra. Therefore, one would hope that 1-cocycles can be studied in terms of the coactions which they produce, or that coactions of the type described above can be examined through the 1-cocycles that they determine. In the rest of this paper, we will pursue this line of research.

In this section, we will compare two important algebraic invariants; the Connes spectrum of a coaction, and the asymptotic range of a 1-cocycle, and show that they actually coincide with each other.

As in $\S 5$, let $c: \mathcal{G} \longrightarrow K$ be a Borel 1-cocycle from a principal measured groupoid $\mathcal{G}$ into a locally compact group $K$. Again we consider the coaction $\alpha_{c}$ of $K$ on the von Neumann algebra $A:=W^{*}(\mathcal{G}, \sigma)$.

Recall that the essential range $\sigma(f)$ of a Borel map $f$ from a measure space $(Y, v)$ into a second countable topological space $Z$ is the smallest closed subset $F$ of $Z$ such that $f^{-1}(F)$ has complement of $v$ measure zero (see just before [9, Definition 8.2]). It is easy to check that $z \in Z$ belongs to $\sigma(f)$ if and only if, for any (compact) neighborhood $U$ of $z$, one has $v\left(f^{-1}(U)\right)>0$. 
Remark. We remark that, for each measurable subset $E$ of $\mathcal{G}$ of positive measure, there exists a measurable subset $F$ of $E$ such that $\chi_{F}$ is a non-zero element of $\mathfrak{A}_{I}$. Indeed, since $v(E)>0$, there exists $E^{\prime} \subseteq E$ such that $0<v\left(E^{\prime}\right)<\infty$. So, there exists $a>1$ such that $0<v\left(E(a) \cap E^{\prime}\right)<\infty$, where $E(a)$ is defined in [15]. Set $F:=E(a) \cap E^{\prime}$. By using [15, Lemma 2.6], we conclude that $\chi_{F}$ is not equal to 0 and belongs to $\mathfrak{A}_{I}$.

In the next lemma, let $H$ be the closed subgroup of $K$ generated by the essential range $\sigma(c)$ of the 1-cocycle $c$. Roughly, the lemma says that we may regard $\alpha=\alpha_{c}$ as a coaction of $H$.

LEMma 6.1. Let the notation be as above. The von Neumann algebra $\lambda_{K}(H)^{\prime \prime}$ coincides with $\left\{\left(\operatorname{id}_{W^{*}(K)} \otimes \omega\right)(\alpha(a)): a \in A, \omega \in A_{*}\right\}^{\prime \prime}$.

To prove the lemma, we use the following.

Lemma 6.2. Let $f \in \mathfrak{A}_{I}$ and $\omega \in A(K)$. Then $\left(\alpha_{c}\right)_{\omega}\left(L^{\sigma}(f)\right):=\left(\omega \otimes \operatorname{id}_{A}\right)\left(\alpha_{c}\left(L^{\sigma}(f)\right)\right)$ equals $L^{\sigma}((\omega \circ c) f)$.

Proof. We may and do assume that $\omega$ has the form $\omega=\omega_{\eta_{1}, \eta_{2}}$ for some $\eta_{1}, \eta_{2} \in L^{2}(K)$. For any $\zeta_{1}, \zeta_{2} \in L^{2}(\mathcal{G})$, by equation (4.1), we have

$$
\begin{aligned}
\left(\left(\alpha_{c}\right)_{\omega}\right. & \left.\left(L^{\sigma}(f)\right) \zeta_{1} \mid \zeta_{2}\right) \\
= & \left(\alpha_{c}\left(L^{\sigma}(f)\right)\left(\eta_{1} \otimes \zeta_{1}\right) \mid \eta_{2} \otimes \zeta_{2}\right) \\
= & \int_{K} \iint \eta_{1}\left(c\left(\gamma_{1}\right)^{-1} k\right) \overline{\eta_{2}(k)} \\
& \times f\left(\gamma_{1}\right) \zeta_{1}\left(\gamma_{1}^{-1} \gamma\right) \sigma\left(\gamma_{1}, \gamma_{1}^{-1} \gamma\right) \overline{\zeta_{2}(\gamma)} d \lambda^{r(\gamma)}\left(\gamma_{1}\right) d \nu(\gamma) d k \\
= & \int \omega\left(c\left(\gamma_{1}\right)\right) f\left(\gamma_{1}\right) \zeta_{1}\left(\gamma_{1}^{-1} \gamma\right) \sigma\left(\gamma_{1}, \gamma_{1}^{-1} \gamma\right) \overline{\zeta_{2}(\gamma)} d \lambda^{r(\gamma)}\left(\gamma_{1}\right) d \nu(\gamma) \\
= & \left(L^{\sigma}((\omega \circ c) f) \zeta_{1} \mid \zeta_{2}\right) .
\end{aligned}
$$

Thus we are done.

Proof of Lemma 6.1. We denote $\left\{\left(\operatorname{id}_{W^{*}(K)} \otimes \omega\right)(\alpha(a)): a \in A, \omega \in A_{*}\right\}^{\prime \prime}$ by $L$.

We first claim that $L \subseteq \lambda_{K}(H)^{\prime \prime}$. If $L$ is not contained in $\lambda_{K}(H)^{\prime \prime}$, there exists $a \in A$ and $\omega \in A_{*}$ such that $\left(\operatorname{id}_{W^{*}(K)} \otimes \omega\right)(\alpha(a)) \notin \lambda_{K}(H)^{\prime \prime}$. We may and do assume that $a=L^{\sigma}(f)$ for some $f \in \mathfrak{A}_{I}$. So there exists $\phi \in A(K)$ such that $\phi\left(\lambda_{K}(H)^{\prime \prime}\right)=0$ and $\phi\left(\left(\operatorname{id}_{W^{*}(K)} \otimes \omega\right) \alpha\left(L^{\sigma}(f)\right)\right) \neq 0$. By Lemma 6.2, we have that $L^{\sigma}((\phi \circ c) f)=$ $\left(\phi \otimes \operatorname{id}_{A}\right)\left(\alpha\left(L^{\sigma}(f)\right)\right)$ is not equal to 0 . In particular, $v(\{\gamma \in \mathcal{G}:(\phi \circ c)(\gamma) f(\gamma) \neq 0\})>0$. On the other hand, by the definition of $\phi$, we obtain $\{\gamma \in \mathcal{G}:(\phi \circ c)(\gamma) f(\gamma) \neq 0\} \cap$ $c^{-1}(H)=\emptyset$. So we conclude that

$$
\begin{aligned}
& v(\{\gamma \in \mathcal{G}:(\phi \circ c)(\gamma) f(\gamma) \neq 0\}) \\
&= v\left(\{\gamma \in \mathcal{G}:(\phi \circ c)(\gamma) f(\gamma) \neq 0\} \cap c^{-1}(H)\right) \\
&+v\left(\{\gamma \in \mathcal{G}:(\phi \circ c)(\gamma) f(\gamma) \neq 0\} \cap c^{-1}(H)^{c}\right) \\
&= 0,
\end{aligned}
$$

a contradiction. Thus our claim has been proved. 
Conversely, we show that $\lambda_{K}(g)$ belongs to $L$ for each $g \in \sigma(c)$. Indeed, if $\lambda_{K}(g)$ is not contained in $L$, then there exists $\phi \in A(K)$ such that $\phi(g) \neq 0$ and $(\phi \otimes \omega) \alpha(a)=0$ for any $\omega \in A_{*}$ and $a \in A$. Since $\phi$ is continuous, there exists a neighborhood $U$ of $g$ such that $\phi\left(\lambda_{K}(h)\right) \neq 0$ for each $h \in U$. By the definition of $\sigma(c), c^{-1}(U)$ is not a $\nu$-null set. So, by the Remark before Lemma 6.1, there exists a measurable subset $E$ of $c^{-1}(U)$ such that $0<v(E)<\infty$ and $\chi_{E} \in \mathfrak{A}_{I}$. Now we set $a:=L^{\sigma}\left(\chi_{E}\right)$. By the definition of $E,(\phi \circ c)(\gamma) \chi_{E}(\gamma) \neq 0$ for each $\gamma \in E$. So, by Lemma 6.2, we have that $\left(\phi \otimes \mathrm{id}_{A}\right) \alpha(a)=L^{\sigma}\left((\phi \circ c) \chi_{E}\right)$ is not equal to 0 . In particular, there exists $\omega \in A_{*}$ such that $(\phi \otimes \omega) \alpha(a) \neq 0$, a contradiction.

Therefore we complete the proof.

According to [9, Definition 8.2], the asymptotic range $r^{*}(c)$ of the 1-cocycle $c$ is by definition $\cap\left\{\sigma\left(c_{B}\right): B \subseteq X, \mu(B)>0\right\}$, where $c_{B}$ stands for the restriction of $c$ to the reduction $\mathcal{G}_{B}$ by $B$.

Our aim of this section is to establish a relationship between $r^{*}(c)$ and $\Gamma\left(\alpha_{c}\right)$.

Let $e$ be a projection in $D=L^{\infty}(X)$ and $B$ be the Borel subset of $X$ satisfying $e=\chi_{B} \circ r$. Then it is easy to see that the reduced von Neumann algebra $e A e$ is (isomorphic to) $W^{*}\left(\mathcal{G}_{B}, \sigma_{B}\right)$. Note that the action $\left(\alpha_{c}\right)^{e}$ obtained by restricting $\alpha_{c}$ to $e A e$ is that derived from the 1-cocycle $c_{B}$.

THEOREM 6.3. We have $\Gamma\left(\alpha_{c}\right)=r^{*}(c)$.

Proof. Since the center $\mathcal{Z}\left(A^{\alpha}\right)$ is contained in $D$, we have

$$
\Gamma\left(\alpha_{c}\right)=\cap\left\{\operatorname{Sp}\left(\left(\alpha_{c}\right)^{e}\right): e: \text { non-zero projection in } D\right\} .
$$

Hence, thanks to the argument preceding this theorem, it suffices to show that $\operatorname{Sp}\left(\alpha_{c}\right)=\sigma(c)$.

Let $k \in \sigma(c)$. Take any compact neighborhood $U$ of $k$. Since $v\left(c^{-1}(U)\right)>0$, by the Remark before Lemma 6.1, there exists a measurable subset $E \subseteq c^{-1}(U)$ such that $v(E)>0$ and $\chi_{E} \in \mathfrak{A}_{I}$. Then define $a:=L^{\sigma}\left(\chi_{E}\right) \in A \backslash\{0\}$. If $\omega \in A(K)$ vanishes on some neighborhood of $U$, then, by Lemma 6.2, we have $\left(\alpha_{c}\right)_{\omega}(a)=0$. From [23, Ch. IV, Lemma 1.2(ii)], it follows that $\operatorname{Sp}_{\alpha_{c}}(a) \subseteq U$. Hence $a$ belongs to $A^{\alpha_{c}}(U)$. By [23, Ch. IV, Lemma 1.2(iv)], $k$ lies in $\operatorname{Sp}\left(\alpha_{c}\right)$.

Conversely, suppose that $k \in \mathrm{Sp}\left(\alpha_{c}\right)$. We will show that for each open neighborhood $V$ of $k, c^{-1}(V)$ is not a $v$-null set. Indeed, if $v\left(c^{-1}(V)\right)$ is equal to 0 for some $V$, we have $L^{\sigma}(f)=L^{\sigma}\left(f \chi_{c^{-1}(V)^{c}}\right)$ for each $f \in \mathfrak{A}_{I}$. So, for each $\omega \in A(K)$ such that supp $\omega \subseteq V$ by Lemma 6.2, we have that

$$
\left(\alpha_{c}\right)_{\omega}\left(L^{\sigma}(f)\right)=L^{\sigma}\left(f \chi_{c^{-1}(V)^{c}}(\omega \circ c)\right)=0 .
$$

So we conclude that $\left(\alpha_{c}\right)_{\omega}(a)=0$ for each $a \in A$ and $\omega \in A(K)$ such that $\operatorname{supp} \omega \subseteq V$. In the meantime, since $V$ is open, for each $h \in V$, there exists $\omega \in A(K)$ such that $\omega(h)=1$ and $\operatorname{supp} \omega \subseteq V$. This shows that for each $a \in A, h \notin \operatorname{Sp}_{\alpha_{c}}(a)$. This contradicts [23, Ch. IV, Lemma 1.2(iv)]. Therefore, $k$ belongs to $\sigma(c)$.

COROllary 6.4. The normalized Connes spectrum $\Gamma_{n}\left(\alpha_{c}\right)$ of $\alpha_{c}$ in the sense of [23, Ch. V, Definition 2.5] coincides with the normalized proper range $\operatorname{npr}(c)$ in the sense of $[9]$. 
By combining Theorem 6.3 with one of the results of Golodets and Sinel'shchikov in [14], we can extend the main result of Kawahigashi in [19] concerning the classification, up to cocycle conjugacy, of actions of locally compact abelian groups on the AFD type II factors which fix Cartan subalgebras.

Proposition 6.5. Let $A$ be an AFD type II factor. Suppose that $\alpha$ and $\alpha^{\prime}$ are coactions of a locally compact group $K$ on $A$ such that each of $A^{\alpha}$ and $A^{\alpha^{\prime}}$ contains a Cartan subalgebra of A. If $\Gamma(\alpha)=\Gamma\left(\alpha^{\prime}\right)=K$, then $\alpha$ is cocycle conjugate to $\alpha^{\prime}$.

Proof. Suppose that $A^{\alpha}$ (respectively $A^{\alpha^{\prime}}$ ) contains a Cartan subalgebra $D_{1}$ (respectively $D_{2}$ ) of $A$. By [5], there exists a $*$-automorphism $\theta$ of $A$ such that $\theta\left(D_{1}\right)=D_{2}$. Set $\alpha_{\theta}:=\left(\operatorname{id}_{W^{*}(K)} \otimes \theta^{-1}\right) \circ \alpha \circ \theta$. Then we have $A^{\alpha_{\theta}}=\theta\left(A^{\alpha}\right)$. So $D_{2}=\theta\left(D_{1}\right) \subseteq \theta\left(A^{\alpha}\right)=A^{\alpha_{\theta}}$. Clearly, $\alpha_{\theta}$ is cocycle conjugate to $\alpha$. Hence it suffices to assume from the outset that $D_{1}=D_{2}=: D$.

We may assume that the inclusion $(D \subseteq A)$ is of the form $\left(L^{\infty}(X) \subseteq W^{*}(\mathcal{R})\right)$ for an amenable ergodic type II equivalence relation $\mathcal{R}$ on a standard Borel space $(X, \mathfrak{B}, \mu)$ with an invariant measure $\mu$. By Theorem 5.8, there exist Borel 1-cocycles $c$ and $c^{\prime}$ from $\mathcal{R}$ to $K$ such that $\alpha=\alpha_{c}$ and $\alpha^{\prime}=\alpha_{c^{\prime}}$. By Theorem 6.3, we have $r^{*}(c)=r^{*}\left(c^{\prime}\right)=K$, i.e. both $\mathcal{R}_{c}$ and $\mathcal{R}_{c^{\prime}}$ are ergodic equivalence relations. So we may apply [14, Lemma 1.13], and obtain that there exist cocycles $\bar{c}$ and $\overline{c^{\prime}}$ cohomologous to $c$ and $c^{\prime}$, respectively, as 1-cocycles on $\mathcal{R}$ such that $\bar{c}$ is equal to $\overline{c^{\prime}} \circ \rho$ for some $\rho \in N[\mathcal{R}]$, the normalizer of $\mathcal{R}$. By Proposition 4.8, $\alpha$ (respectively $\alpha^{\prime}$ ) is cocycle conjugate to $\alpha_{\bar{c}}$ (respectively $\alpha_{\overline{c^{\prime}}}$ ). Furthermore, a direct computation shows that for each $X \in W^{*}(\mathcal{R})$,

$$
\alpha_{\bar{c} \circ \theta}(X)=\left(1 \otimes \Phi_{\rho}^{-1}\right)\left(\alpha_{\bar{c}}\left(\Phi_{\rho}(X)\right)\right),
$$

where $\Phi_{\rho}$ is an automorphism on $W^{*}(\mathcal{R})$ that is defined by

$$
\Phi_{\rho}(L(f)):=L(f \circ \rho) .
$$

So we conclude that $\left(1 \otimes \Phi_{\rho}\right) \alpha_{\bar{c} \circ \rho}=\alpha_{\bar{c}} \circ \Phi_{\rho}$, i.e. $\alpha_{\bar{c} \circ \rho}$ is conjugate to $\alpha_{\bar{c}}$. Hence $\alpha$ is cocycle conjugate to $\alpha^{\prime}$.

We note that, by using the notion of a double cocycle in the sense of Golodets and Sinel'shchikov [14], it is possible to classify those coactions on (not necessarily type II) AFD factors which fix Cartan subalegbras. Assume that $\mathcal{R}$ is an amenable ergodic equivalence relation. Let $c$ and $c^{\prime}$ be non-transient Borel 1-cocycles on $\mathcal{R}$ to a locally compact group $K$. Set $A:=W^{*}(\mathcal{R})$ and $r:=\log \delta$. By [14, Theorem 3.1], we see that, if the double cocycles $c \times r$ and $c^{\prime} \times r$ are conjugate, then $c$ is weakly equivalent to $c^{\prime}$. Meanwhile, one can check that the crossed product $\widehat{K \times \mathbf{R}} \alpha_{c \times r} \ltimes A$ is isomorphic to the crossed product of $\widehat{K}_{\alpha_{c}} \ltimes A$ by a modular action. Therefore, Theorem 3.1 in [14] can be translated in an operator-algebraic term as follows.

THEOREM 6.6. Let $\alpha$ and $\alpha^{\prime}$ be coactions of a locally compact group $K$ on an AFD factor $A$ such that each of $A^{\alpha}$ and $A^{\alpha^{\prime}}$ contains a Cartan subalgebra of $A$. Suppose that the crossed products $P_{1}:=\widehat{K}_{\alpha} \ltimes A$ and $P_{2}:=\widehat{K}_{\alpha^{\prime}} \ltimes A$ are non-type I von Neumann algebras. Then $\alpha$ is cocycle conjugate to $\alpha^{\prime}$ if and only if there exists a $*$-isomorphism $\Pi$ from the center $\mathcal{Z}_{1}$ of the crossed product $\mathbf{R} \ltimes P_{1}$ by a modular action on $P_{1}$ onto the center $\mathcal{Z}_{2}$ of the 
crossed product $\mathbf{R} \ltimes P_{2}$ by a modular action on $P_{2}$ such that, with $\theta^{(i)} \quad(i=1,2)$ the dual action of the modular action on $P_{i}$, and with $(\widetilde{\widetilde{\alpha}})$ (respectively $\left.\left(\widetilde{\widetilde{\alpha^{\prime}}}\right)\right)$ the canonical extension of the dual action $\widehat{\alpha}$ (respectively $\widehat{\alpha^{\prime}}$ ) of $\alpha$ (respectively $\alpha^{\prime}$ ) to $\mathbf{R} \ltimes P_{1}$ (respectively $\mathbf{R} \ltimes P_{2}$ ), $\Pi$ intertwines the product actions $\theta^{(1)}\left|\mathcal{Z}_{1} \circ(\widetilde{\widetilde{\alpha}})_{k}\right|_{\mathcal{Z}_{1}}$ of $K \times \mathbf{R}$ on $\mathcal{Z}_{1}$ and $\theta^{(2)}\left|\mathcal{Z}_{2} \circ\left(\widetilde{\widetilde{\alpha^{\prime}}}\right)_{k}\right| \mathcal{Z}_{2}$ on $\mathcal{Z}_{2}$.

We conclude this section with a result on spectral subspaces for the case of $\mathcal{G}$ being a discrete principal measured groupoid. Recall that, for each closed subset $H$ of $K$, the spectral subspace $A^{\alpha}(H)$ is defined by

$$
A^{\alpha}(H):=\left\{a \in A: \operatorname{Sp}_{\alpha}(a) \subseteq H\right\} .
$$

It is easy to see that $A^{\alpha}(H)$ is a bimodule over the Cartan subalgebra $D$. In what follows, we prove that $A^{\alpha}(H)$ is determined by a Borel subset of $\mathcal{G}$. For this purpose, we use the following lemma.

LEMMA 6.7. Suppose that an element $\rho$ of the full group $[\mathcal{G}]_{*}$ of $\mathcal{G}$ satisfies $v(\Gamma(\rho))>0$, and that $c(\Gamma(\rho))$ is contained in open subset $L$ of $K$. Here $\Gamma(\rho)$ stands for the graph of $\rho$. Then there exists $k \in L$ such that $v\left(\Gamma(\rho) \cap c^{-1}(U)\right)>0$ for any neighborhood $U$ of $k$.

Proof. Suppose that, for each $k \in L$, there exists a neighborhood $U_{k}$ of $k$ such that $v\left(\Gamma(\rho) \cap c^{-1}\left(U_{k}\right)\right)=0$. Since $\left\{U_{k}\right\}_{k \in L}$ is a covering of the open set $L$, there exists a sequence $\left\{k_{n}\right\}_{n=1}^{\infty}$ in $L$ such that $\left\{U_{k_{n}}\right\}_{n=1}^{\infty}$ is a covering of $L$. So we have

$$
v(\Gamma(\rho)) \leq \sum_{n=1}^{\infty} v\left(\Gamma(\rho) \cap c^{-1}\left(U_{k_{n}}\right)\right)=0,
$$

which is, a contradiction.

PROPOSITION 6.8. Let the notation be as above. The spectral subspace $A^{\alpha}(H)$ is equal to $\mathfrak{I}\left(c^{-1}(H)\right)$, where $\mathfrak{I}\left(c^{-1}(H)\right)$ is defined by the following expression:

$$
\Im\left(c^{-1}(H)\right):=\left\{L^{\sigma}(f) \in A: v\left(\operatorname{supp}(f) \cap c^{-1}(H)^{c}\right)=0\right\} .
$$

Proof. It is easy to check that $\mathfrak{I}\left(c^{-1}(H)\right)$ is contained in $A^{\alpha}(H)$.

Conversely, if $L^{\sigma}(f)$ is in $A^{\alpha}(H) \backslash \Im\left(c^{-1}(H)\right)$, then there exists $\rho \in[\mathcal{G}]_{*}$ such that $\Gamma(\rho) \subseteq \mathcal{R} \backslash c^{-1}(H)$ and $v(\operatorname{supp}(f) \cap \Gamma(\rho))>0$. By using the previous lemma, we may assume that $c(\Gamma(\rho))$ is contained in a compact subset $U$ of $K \backslash H$. Now we set $v:=L^{\sigma}\left(\chi_{\Gamma(\rho)} \delta^{-1 / 2}\right)$ and $a:=E\left(L^{\sigma}(f) v^{*}\right) v$, where $E: A \rightarrow D$ is the faithful normal conditional expectation. Since $A^{\alpha}(H)$ is a bimodule over $D, a$ is a non-zero element of $A^{\alpha}(H)$. However, by the construction of $a$, for each $h \in H$, there exists $\phi_{h} \in A(K)$ such that $\phi_{h}(h)=1$ and $\operatorname{supp}(\phi) \cap U=\emptyset$, i.e. $h$ does not belong to $\operatorname{Sp}_{\alpha}(a)$. So we conclude $H \cap \operatorname{Sp}_{\alpha}(a)=\emptyset$, i.e. $a \notin A^{\alpha}(H)$, is a contradiction.

Therefore we complete the proof.

7. Exchangeability for a 1-cocycle with a smaller range within the cohomology class It sometimes happens that a Borel 1-cocycle on a measured groupoid into a group can be exchanged, within its cohomology class, for a cocycle whose range is a smaller subgroup. 
In this section, we choose to study this phenomenon from a (operator-algebraic) viewpoint, as taken in the previous sections. It should be noted that a study from a measure-theoretic viewpoint was taken up by Zimmer in [37] when a group in question is compact.

Let $\mathcal{R}$ be a discrete measured equivalence relation on a standard Borel probability measure space $(X, \mathfrak{B}, \mu), \sigma$ a Borel 2-cocycle on $\mathcal{R}$ and $c: \mathcal{R} \rightarrow K$ be a Borel 1-cocycle on $\mathcal{R}$. We set $A:=W^{*}(\mathcal{R}, \sigma)$ and denote by $D$ the associated Cartan subalgebra $L^{\infty}(X, \mu)$ of $A$. Let $\alpha_{c}$ be the coaction of $K$ on $A$ determined by $c$. We will freely use the notation introduced in $\$ \S 2$ and 3 .

Suppose first that $c$ is cohomologous to a Borel 1-cocycle $c^{\prime}$ whose range is contained in a closed subgroup $H$ of $K$. As we saw before, there is a unitary $R \in W^{*}(K) \otimes D$ satisfying $\alpha_{c^{\prime}}=\operatorname{Ad} R \circ \alpha_{c}$ (in particular, $R$ is an $\alpha_{c^{-1}}$-cocycle). Hence the corresponding two dual-covariant systems $\left(\tilde{A}:=\widehat{K}_{\alpha_{c}} \ltimes A, K, \widehat{\alpha_{c}}\right),\left(\bar{A}:=\widehat{K}_{\alpha_{c^{\prime}}} \ltimes A, K, \widehat{\alpha_{c^{\prime}}}\right)$ are conjugate. Consequently, $\left(\mathcal{Z}(\tilde{A}), K,\left.\widehat{\alpha_{c}}\right|_{\mathcal{Z}(\tilde{A})}\right)$ and $\left(\mathcal{Z}(\bar{A}), K,\left.\widehat{\alpha_{c^{\prime}}}\right|_{\mathcal{Z}(\bar{A})}\right)$ (i.e. the Mackey actions) are conjugate. We know that

$$
\begin{gathered}
\mathcal{Z}(\bar{A})=\left\{F \in L^{\infty}(K \times X): F(k, x)=F\left(c^{\prime}(y, x) k, y\right)\right. \\
\left.\left(\text { almost every }(k,(x, y)) \in K \times{ }_{c^{\prime}} \mathcal{R}\right)\right\}
\end{gathered}
$$

Since the range of $c^{\prime}$ is contained in $H$, the map $\tau: L^{\infty}(K / H) \rightarrow \mathcal{Z}(\bar{A})$ given by $\tau(\phi):=\phi^{\vee} \otimes 1 \quad\left(\phi \in L^{\infty}(K / H)\right)$ is well defined, where $f^{\vee}(k):=f\left(k^{-1}\right)$ for $f \in L^{\infty}(K)$. (Note that in what follows, we freely identify a function $f$ in $L^{\infty}(K / H)$ with a function in $L^{\infty}(K)$ that satisfies $f(k h)=f(k)$ for all $h \in H$.) It is also easy to see that $\tau$ is a $K$-equivariant embedding of $L^{\infty}(K / H)$ into $\mathcal{Z}(\bar{A})$. Hence, from [31] (see also Appendix A), we find that the dual action $\widehat{\alpha_{c^{\prime}}}$ is induced from an action $\beta$ of $H$ on a von Neumann algebra $P$. Our immediate purpose is to identify this covariant system $(P, H, \beta)$. For this, let us denote $c^{\prime}$ by $c_{0}$ when $c^{\prime}$ is viewed as a 1-cocycle from $\mathcal{R}$ into $H$. So $\alpha_{c_{0}}$ is a coaction of $H$ on $A$. Put $P:=\widehat{H}_{\alpha_{c_{0}}} \ltimes A$ and $\beta:=\widehat{\alpha_{c_{0}}}$.

From now on, we fix a quasi-invariant measure $\Omega$ on $K / H$. As in Appendix A, denote by $\rho$ the rho-function on $K$ associated with $\mu$. We choose a Borel cross section $\theta: K / H \rightarrow K$ of the quotient map $\pi: K \rightarrow K / H$ with $\theta(\pi(e))=e$.

Define three unitaries $T_{K}=T_{K}^{*}$ from $L^{2}(K)$ onto itself, $V$ from $L^{2}(K)$ onto $L^{2}(K / H) \otimes L^{2}(H)$ and $T_{H}=T_{H}^{*}$ on $L^{2}(H)$ by

$$
\begin{gathered}
\left\{T_{K} f\right\}(k):=\Delta_{K}(k)^{-1 / 2} f\left(k^{-1}\right) \quad\left(f \in L^{2}(K), k \in K\right), \\
\{V \xi\}(p, h):=\xi(\theta(p) h) \rho(\theta(p) h)^{-1 / 2} \quad\left(\xi \in L^{2}(K), p \in K / H, h \in H\right), \\
\left\{V^{*} \eta\right\}(k)=\eta\left(\pi(k), h_{k}\right) \rho(k)^{1 / 2} \quad\left(\eta \in L^{2}(K / H \times H)\right), \\
\left\{T_{H} f\right\}(h):=\Delta_{H}(h)^{-1 / 2} f\left(h^{-1}\right) \quad\left(f \in L^{2}(H), h \in H\right) .
\end{gathered}
$$

Note that $T_{K} \lambda_{K}(k) T_{K}=\rho_{K}(k)$ and $T_{H} \lambda_{H}(h) T_{H}=\rho_{H}(h)$. We also have $V^{*}(\phi \otimes 1) V=$ $\phi\left(\phi \in L^{\infty}(K / H)\right), V^{*}\left(L^{\infty}(K / H) \otimes L^{\infty}(H)\right) V=L^{\infty}(K)$ and $V^{*}\left(1 \otimes \rho_{H}(h)\right) V=$ $\rho_{K}(h)$ for all $h \in H$. 
Set $S_{1}:=\left(V^{*} \otimes 1_{L^{2}(\mathcal{R})}\right)\left(1_{L^{2}(K / H)} \otimes T_{H} \otimes 1_{L^{2}(\mathcal{R})}\right)$. Then we have

$$
\begin{aligned}
& S_{1}\left(L^{\infty}(K / H) \otimes P\right) S_{1}^{*} \\
& \quad=S_{1}\left\{\left(L^{\infty}(K / H) \otimes L^{\infty}(H) \otimes 1_{L^{2}(\mathcal{R})}\right) \vee\left(1_{L^{2}(K / H)} \otimes \alpha_{c_{0}}(A)\right)\right\} S_{1}^{*} \\
& \quad=\left(L^{\infty}(K) \otimes 1_{L^{2}(\mathcal{R})}\right) \vee S_{1}\left(1_{L^{2}(K / H)} \otimes \alpha_{c_{0}}(A)\right) S_{1}^{*} .
\end{aligned}
$$

Next we set $S_{2}:=\left(T_{K} \otimes 1_{L^{2}(\mathcal{R})}\right) S_{1}$. From the above computation, we get

$$
S_{2}\left(L^{\infty}(K / H) \otimes P\right) S_{2}^{*}=\left(L^{\infty}(K) \otimes 1_{L^{2}(\mathcal{R})}\right) \vee S_{2}\left(1_{L^{2}(K / H)} \otimes \alpha_{c_{0}}(A)\right) S_{2}^{*} .
$$

By a direct computation using equation (4.1), we see that

$$
S_{2}\left(1_{L^{2}(K / H)} \otimes \alpha_{c_{0}}\left(L^{\sigma}(f)\right)\right) S_{2}^{*}=\alpha_{c^{\prime}}\left(L^{\sigma}(f)\right) .
$$

Hence

$$
S_{2}\left(L^{\infty}(K / H) \otimes P\right) S_{2}^{*}=\bar{A} .
$$

Moreover, by using properties of the $\rho$-function (cf. $[11, \S 2.6])$ and the notation described in the Appendix A, one can verify that

$$
\begin{aligned}
& \left\{S_{2}^{*}\left(\rho_{K}(k) \otimes 1_{L^{2}(\mathcal{R})}\right) S_{2} \eta\right\}(p, h,(x, y)) \\
& \quad=\left[\frac{d \Omega \circ k^{-1}}{d \Omega}(p)\right]^{1 / 2} \Delta_{H}\left(\chi\left(k^{-1}, p\right)\right)^{-1 / 2} \eta\left(k^{-1} p, h \chi\left(k^{-1}, p\right)^{-1},(x, y)\right)
\end{aligned}
$$

for any $\eta \in L^{2}(K / H \times H \times \mathcal{R})$. From this, we find that $\operatorname{Ad} S_{2}^{*}\left(\rho_{K}(\cdot) \otimes 1_{L^{2}(\mathcal{R})}\right) S_{2}$ is exactly the action $\delta$ of $K$ defined in the Appendix A. We summarize the results obtained so far in the proposition that follows.

PROPOSITION 7.1. Let $\mathcal{R}$ be a discrete measured equivalence relation on a standard Borel probability measure space $(X, \mathfrak{B}, \mu), \sigma$ a Borel 2-cocycle on $\mathcal{R}$ and $c: \mathcal{R} \rightarrow K$ be a Borel 1-cocycle on $\mathcal{R}$ into a locally compact group $K$. We set $A:=W^{*}(\mathcal{R}, \sigma)$. Let $\alpha_{c}$ be the coaction of $K$ on A determined by $c$.

If $c$ is cohomologous to a Borel 1-cocycle $c^{\prime}$ whose range is contained in a closed subgroup $H$ of $K$, then the dual action $\widehat{\alpha_{c}}$ is induced from an action $\beta$ of $H$ on $a$ von Neumann algebra $P$. In this case, one can take the covariant system $(P, H, \beta)$ to be $\left(\widehat{H}_{\alpha_{c_{0}}} \ltimes A, H, \widehat{\alpha_{c_{0}}}\right)$, where $c_{0}: \mathcal{R} \rightarrow H$ is the 1-cocycle obtained by regarding $c^{\prime}$ as an $H$-valued 1-cocycle.

So far, we have seen that the fact that a given 1-cocycle can be exchanged for another 1-cocycle whose range is contained in a smaller subgroup implies that the dual action of the associated coaction is induced from an action of the smaller subgroup. As we will see below, these conditions are in fact equivalent.

THEOREM 7.2. Let $\mathcal{R}$ and $K$ be as before. Suppose that $c: \mathcal{R} \rightarrow K$ is a Borel 1-cocycle, and let $\alpha_{c}$ be the corresponding coaction of $K$ on $A:=W^{*}(\mathcal{R}, \sigma)$. We also let $H$ be a closed subgroup of $K$. Then the following assertations are equivalent.

(1) There exists a Borel 1-cocycle $c^{\prime}: \mathcal{R} \rightarrow K$, cohomologous to $c$, such that the range of $c^{\prime}$ is contained in $H$. 
(2) There exists an injective normal $*$-homomorphism $\Theta$ from $L^{\infty}(K / H)$ into the center of the crossed product $\widehat{K}_{\alpha_{c}} \ltimes A$ such that $\Theta \circ \ell_{k}={\widehat{\left(\alpha_{c}\right)_{k}}}_{0} \odot$ for all $k \in K$, where $\ell_{k}$ is the action derived from the left translation by $k$ on the homogeneous space $K / H$. (3) The dual system $\left\{\widehat{K}_{\alpha_{c}} \ltimes A, K, \widehat{\alpha_{c}}\right\}$ is induced from some covariant system $\{P, H, \beta\}$. If one of (1)-(3) occurs, then one can take $\{P, H, \beta\}$ to be $\left\{\widehat{H}_{\alpha_{c_{0}}} \ltimes A, H, \widehat{\alpha_{c_{0}}}\right\}$, where $c_{0}: \mathcal{R} \rightarrow H$ is the 1 -cocycle obtained by regarding $c^{\prime}$ as an $H$-valued 1 -cocycle.

Proof. We have already shown that (1) implies (2). The last half of our assertion is exactly the content of Proposition 7.1. That (2) is equivalent to (3) is exactly the Imprimitivity theorem for induced actions. Therefore, it remains to prove that (2) implies (1). The way this is done is essentially the same as in [37, Theorem 3.5].

As before, let $\tilde{A}:=\widehat{K}_{\alpha_{c}} \ltimes A$. Since $L^{\infty}(K) \otimes D$ is a Cartan subalgebra of $\tilde{A}, \mathcal{Z}(\tilde{A})$ is contained in $L^{\infty}(K) \otimes D=L^{\infty}(K \times X)$, the morphism $\Theta$ can be viewed as a $K$-embedding into $L^{\infty}(K \times X)$. Hence we may assume that there exists a $K$-equivariant Borel map $\psi$ from $K \times X$ onto $K / H$ such that $\Theta(f)=f \circ \psi$. As the range of $\Theta$ is contained in $\mathcal{Z}(\tilde{A})$, we have

$$
\psi(k, x)=\psi\left(c(x, y)^{-1} k, y\right) \quad\left(\text { almost every }(k,(x, y)) \in K \times_{c} \mathcal{R}\right) .
$$

Hence $K_{0}:=\left\{k \in K: \psi(k, x)=\psi\left(c(x, y)^{-1} k, y\right)\right.$ (almost every $\left.\left.(x, y) \in \mathcal{R}\right)\right\}$ is a conull Borel subset of $K$. Thus there exists $k_{0} \in K_{0}$ such that $\mathcal{R}_{0}:=\left\{(x, y) \in \mathcal{R}: \psi\left(k_{0}, x\right)=\right.$ $\left.\psi\left(c(x, y)^{-1} k_{0}, y\right)\right\}$ is conull. So, if $(x, y) \in \mathcal{R}_{0}$, then, because $\psi$ is a $K$-equivariant, we have

$$
k_{0}^{-1} \cdot \psi(e, x)=k_{0}^{-1} c(x, y) \cdot \psi(e, y) .
$$

Equivalently, for all $(x, y) \in \mathcal{R}_{0}$, one has

$$
\psi(e, x)=c(x, y) \cdot \psi(e, y) .
$$

Choose a Borel cross section $\theta: K / H \rightarrow K$ with $\theta(\pi(e))=e$. Then, by equation (7.3), we have

$$
\theta(\psi(e, x))^{-1} c(x, y) \theta(\psi(e, y)) \in H
$$

for all $(x, y) \in \mathcal{R}_{0}$. Motivated by this, we define a new Borel 1-cocycle $c_{1}: \mathcal{R} \rightarrow K$ by

$$
c_{1}(x, y):=\theta(\psi(e, x))^{-1} c(x, y) \theta(\psi(e, y)) \quad((x, y) \in \mathcal{R}) .
$$

By definition, $c_{1}$ is cohomologous to $c$, and $c_{1}(x, y) \in H$ almost every $(x, y)$ by equation (7.4).

Finally we replace $c_{1}$ by another cocycle $c^{\prime}$ that satisfies $c_{1}=c^{\prime}$ almost everywhere and $c^{\prime}(x, y) \in H$ for all $(x, y) \in \mathcal{R}$. For this, put $\mathcal{N}:=\left\{(x, y) \in \mathcal{R}: c_{1}(x, y) \notin H\right\}$. Since $\mathcal{N}$ is a Borel null subset, there exists a Borel null subset $N_{1}$ of $X$ such that, if $x \in\left(N_{1}\right)^{c}$, then $(x, y) \notin \mathcal{N}$, i.e. $c_{1}(x, y) \in H$ for all $y \sim x$. Suppose that $G$ is a countable discrete group acting non-singularly on $X$ that generates the equivalence relation $\mathcal{R}$ [9]. Then set $N:=\bigcup_{g \in G} g N_{1}$, the saturation of $N_{1}$. Note that, by definition, for $(x, y) \in \mathcal{R}$, $x \in N$ if and only if $y \in N$. It is clear that $\mathcal{R} \cap(N \times N)$ is a Borel null subset of $\mathcal{R}$ and $\mathcal{R} \cap(\mathcal{R} \cap(N \times N))^{c}=\mathcal{R} \cap\left(N^{c} \times N^{c}\right)$. Remark also that, if $(x, y) \in \mathcal{R} \cap\left(N^{c} \times N^{c}\right)$, 
then $(x, y) \notin \mathcal{N}$, so that $c_{1}(x, y) \in H$. With these in mind, we define a Borel map $c^{\prime}: \mathcal{R} \rightarrow K$ by

$$
c^{\prime}(x, y)= \begin{cases}c_{1}(x, y) & \text { if }(x, y) \in \mathcal{R} \cap\left(N^{c} \times N^{c}\right), \\ e & \text { if }(x, y) \in \mathcal{R} \cap(N \times N) .\end{cases}
$$

Hence $c^{\prime}=c_{1}$ almost everywhere. It is easy to check that $c^{\prime}$ is a 1-cocycle. Moreover, it is obvious by definition that $c^{\prime}(x, y) \in H$ for all $(x, y) \in \mathcal{R}$. Thus we are done.

We conclude this section with a result on regular cocycles. We recall that a cocycle $c$ from an ergodic discrete equivalence relation $\mathcal{R}$ into a locally compact group $K$ is regular if the Mackey action of $c$ is (essentially) transitive. In [28, Proposition 2.1(2)], Schmidt claimed that $c$ is regular if and only if there exists a 1-cocycle $c^{\prime}$ cohomologous to $c$ such that the closed subgroup generated by the range of $c^{\prime}$ is equal to $r^{*}\left(c^{\prime}\right)$. However, he did not give a proof to the claim in the paper. Moreover, the paper [27], which is referred to in [28, Proposition 2.1], treated only the case of the group $K$ being abelian. So, in this paper, we will give a complete proof to a more general situation.

Definition 7.3. Let $\left(\mathcal{R},\left\{\lambda^{x}\right\}_{x \in X}, \mu\right)$ be a principal measured groupoid and $K$ be a locally compact group. We say that a Borel 1-cocycle $c: \mathcal{R} \rightarrow K$ is regular if there exists a 1-cocycle $c^{\prime}$ cohomologous to $c$ such that the closed subgroup generated by the range of $c^{\prime}$ is equal to $r^{*}\left(c^{\prime}\right)$.

Proposition 7.4. Suppose that $c: \mathcal{R} \rightarrow K$ is a Borel 1-cocycle from a discrete measured equivalence relation $\mathcal{R}$ on $(X, \mu)$ into a locally compact group $K$. Let $A$ be the groupoid von Neumann algebra of $\mathcal{R}$, twisted by a Borel 2-cocycle on $\mathcal{R}$. The following assertations are equivalent:

(1) the cocycle $c$ is regular.

(2) there exists a closed subgroup $H$ of $K$ such that each ergodic component of the Mackey action of $c$ is $K$-isomorphic to $K / H$, i.e. the covariant system $\left(\mathcal{Z}\left(\widehat{K}_{\alpha_{c}} \ltimes A\right), K,\left.\widehat{\alpha_{c}}\right|_{\mathcal{Z}\left(\widehat{K}_{\alpha_{c}} \ltimes A\right)}\right)$ is conjugate to $\left(L^{\infty}(K / H) \otimes \mathcal{Z}(A), K, \ell_{k} \otimes\right.$ $\left.\operatorname{id}_{\mathcal{Z}(A)}\right)$, where $\ell_{k}$ is as in Theorem 7.2.

In particular, if $\left.\widehat{\alpha_{c}}\right|_{\mathcal{Z}\left(\widehat{K}_{\alpha_{c}} \ltimes A\right)}$ is transitive on $\mathcal{Z}\left(\widehat{K}_{\alpha_{c}} \ltimes A\right)$, (which implies that $A$ is a factor) then $c$ is regular.

Proof. Put $\tilde{A}:=\widehat{K}_{\alpha_{c}} \ltimes A$.

$(1) \Rightarrow(2)$ : Since $c$ is regular, there exists a 1-cocycle $c^{\prime}$ cohomologous to $c$ such that the asymptotic range of $c^{\prime}$ is generated by the range of $c^{\prime}$. Set $H=r^{*}\left(c^{\prime}\right)$. As before, denote by $c_{0}$ the cocycle $c^{\prime}$ viewed as a cocycle from $\mathcal{R}$ into $H$. By definition, we have $\Gamma\left(\alpha_{c_{0}}\right)=r^{*}\left(c_{0}\right)=H$. By [23, Ch. IV, Corollary 1.6] and [23, Ch. V, Corollary 2.7], we conclude that the center of $\widehat{H}_{\alpha_{c_{0}}} \ltimes A$ is equal to $\alpha_{c_{0}}(\mathcal{Z}(A))$.

In the meantime, by Proposition 7.1, $\widehat{K}_{\alpha_{c^{\prime}}} \ltimes A$ is isomorphic to $L^{\infty}(K / H) \otimes \widehat{H}_{\alpha_{c_{0}}} \ltimes A$, and the dual action $\widehat{\alpha_{c^{\prime}}}$ is conjugate, through this isomorphism, to the action $\delta$ of $K$ defined in the Appendix A. By the previous paragraph, we have $\mathcal{Z}\left(L^{\infty}(K / H) \otimes \widehat{H}_{\alpha_{c_{0}}} \ltimes A\right)=$ $L^{\infty}(K / H) \otimes \alpha_{c_{0}}(\mathcal{Z}(A))$. It is easy to verify that, on this abelian subalgebra, the action $\delta_{k}$ is exactly $\ell_{k} \otimes \operatorname{id}_{\alpha_{c_{0}}}(\mathcal{Z}(A))$. Thus we are done. 
$(2) \Rightarrow(1)$ : By assumption, there is a $*$-isomorphism $\Phi$ from $\mathcal{Z}(\tilde{A})$ onto $L^{\infty}(K / H) \otimes$ $\mathcal{Z}(A)$ such that $\Phi \circ{\widehat{\left(\alpha_{c}\right)_{k}}}_{k}\left(\ell_{k} \otimes \operatorname{id}_{\mathcal{Z}(A)}\right) \circ \Phi$ for all $k \in K$. Then $\Theta(f):=\Phi^{-1}(f \otimes 1)$ $\left(f \in L^{\infty}(K / H)\right)$ defines an injective normal $*$-homomorphism from $L^{\infty}(K / H)$ into $\mathcal{Z}(\tilde{A})$ satisfying $\Theta \circ \ell_{k}=\widehat{\left(\alpha_{c}\right)_{k}} \circ \Theta$ for any $k \in K$. By Theorem 7.2, we have that: (i) there exists a Borel 1-cocycle $c^{\prime}$ cohomologous to $c$ such that the range of $c^{\prime}$ is contained in $H$; (ii) there is a $K$-isomorphism $\Psi$ from $\widehat{K}_{\alpha_{c}} \ltimes A$ onto $L^{\infty}(K / H) \otimes \widehat{H}_{\alpha_{c_{0}}} \ltimes A$, where $c_{0}$ is, as before, the 1-cocycle obtained by regarding $c^{\prime}$ as an $H$-valued 1-cocycle. Moreover, by the construction, we may assume that $\Psi$ satisfies the equation $\Psi^{-1}(f \otimes 1)=\Theta(f)$ for all $f \in$ $L^{\infty}(K / H)$. Denote by $\delta$ (as in Appendix A) the action of $K$ on $L^{\infty}(K / H) \otimes \widehat{H}_{\alpha_{c_{0}}} \ltimes A$, conjugate to $\widehat{\alpha_{c}}$ through $\Psi$.

Put $\Pi:=\left.\Phi \circ \Psi^{-1}\right|_{L^{\infty}(K / H) \otimes \mathcal{Z}\left(\widehat{H}_{\alpha_{c_{0}}} \ltimes A\right)}$. Since $\Pi \circ \delta_{k}=\left(\ell_{k} \otimes \mathrm{id}\right) \circ \Pi$ for all $k \in K$, we have

$$
\Pi\left(\left(L^{\infty}(K / H) \otimes \mathcal{Z}\left(\widehat{H}_{\alpha_{c_{0}}} \ltimes A\right)\right)^{\delta}\right)=\left(L^{\infty}(K / H) \otimes \mathcal{Z}(A)\right)^{\ell \otimes \operatorname{id} \mathcal{Z}(A)}=\mathbf{C} \otimes \mathcal{Z}(A) .
$$

Meanwhile, because

$$
\left(L^{\infty}(K / H) \otimes \widehat{H}_{\alpha_{c_{0}}} \ltimes A\right)^{\delta}=\mathbf{C} \otimes\left(\widehat{H}_{\alpha_{c_{0}}} \ltimes A\right)^{\widehat{\alpha_{c_{0}}}}=\mathbf{C} \otimes \alpha_{c_{0}}(A),
$$

it follows that $\left(L^{\infty}(K / H) \otimes \mathcal{Z}\left(\widehat{H}_{\alpha_{c_{0}}} \ltimes A\right)\right)^{\delta}=\mathbf{C} \otimes \alpha_{c_{0}}(\mathcal{Z}(A))$. Hence we get

$$
\Pi\left(\mathbf{C} \otimes \alpha_{c_{0}}(\mathcal{Z}(A))\right)=\mathbf{C} \otimes \mathcal{Z}(A) .
$$

We also know that

$\Pi\left(L^{\infty}(K / H) \otimes \mathbf{C}\right)=\Phi\left(\Psi^{-1}\left(L^{\infty}(K / H) \otimes \mathbf{C}\right)\right)=\Phi\left(\Theta\left(L^{\infty}(K / H)\right)\right)=L^{\infty}(K / H) \otimes \mathbf{C}$.

Hence we have

$$
\begin{aligned}
\Pi\left(L^{\infty}(K / H) \otimes \mathcal{Z}\left(\widehat{H}_{\alpha_{c_{0}}} \ltimes A\right)\right) & =L^{\infty}(K / H) \otimes \mathcal{Z}(A) \\
& =\left(L^{\infty}(K / H) \otimes \mathbf{C}\right) \vee(\mathbf{C} \otimes \mathcal{Z}(A)) \\
& =\Pi\left(L^{\infty}(K / H) \otimes \mathbf{C}\right) \vee \Pi\left(\mathbf{C} \otimes \alpha_{c_{0}}(\mathcal{Z}(A))\right) \\
& =\Pi\left(L^{\infty}(K / H) \otimes \alpha_{c_{0}}(\mathcal{Z}(A))\right) .
\end{aligned}
$$

From this, it follows that $\mathcal{Z}\left(\widehat{H}_{\alpha_{c_{0}}} \ltimes A\right)=\alpha_{c_{0}}(\mathcal{Z}(A))$. From [23, Ch. IV, Corollary 1.6] and [23, Ch. V, Corollary 2.7] once again, we see that the Connes spectrum of $\alpha_{c_{0}}$ is equal to $H$, i.e. the asymptotic range $r^{*}\left(c_{0}\right)$ coincides with $H$. This in turn implies that $r^{*}\left(c^{\prime}\right)=H$. Therefore, $c$ is a regular cocycle.

\section{Integrability of coactions}

In this section, we will discuss the integrability of the coactions of the type considered in the previous sections.

Let $\alpha$ be a coaction of a locally compact group $K$ on a von Neumann algebra $A$ having a Cartan subalgebra $D$ inside $A^{\alpha}$. As before, we realize $A$ as $W^{*}(\mathcal{R}, \sigma)$ for some discrete measured equivalence relation $\mathcal{R}$ on a standard measure space $(X, \mu)$ and some normalized Borel 2-cocycle $\sigma$ on $\mathcal{R}$. Also choose a Borel 1-cocycle $c: \mathcal{R} \rightarrow K$ in such a way that $\alpha=\alpha_{c}$. Denote by $U_{c}$ the canonical implementation of $\alpha$. We assume that $\mu$ is a (quasiinvariant) probability measure. Then the characteristic function $\xi_{0}$ of the diagonal set of $\mathcal{R}$ 
belongs to $L^{2}(\mathcal{R})$ and is a cyclic and separating vector for $A$. Denote by $J_{\xi_{0}}$ the modular conjugation associated to the faithful normal state $\omega_{\xi_{0}}$ on $A$. Let $A_{2}:=J_{\xi_{0}}\left(A^{\alpha}\right)^{\prime} J_{\xi_{0}}$, the basic extension of the inclusion $A^{\alpha} \subseteq A$. Since $A^{\alpha}$ is $W^{*}\left(\mathcal{S},\left.\sigma\right|_{\mathcal{S}}\right)$ with $\mathcal{S}:=\operatorname{Ker}(c)$, we know that $A_{2}$ is generated by $A$ and the (Jones) projection $\chi_{\mathcal{S}}$.

Suppose now that $\alpha$ is integrable. Then, by [33, Theorem 5.3], there exists a normal and surjective $*$-homomorphism $\rho$ from $\widehat{K}_{\alpha} \ltimes A$ onto $A_{2}$ satisfying

$$
\begin{aligned}
\rho(\alpha(a)) & =a \quad(\forall a \in A), \\
\rho(\omega \otimes 1) & =\omega \circ c \quad(\forall \omega \in A(K)) .
\end{aligned}
$$

Then, for any $\omega \in A(K)$, we have

$$
\rho(\omega \otimes 1) \chi_{\mathcal{S}}=(\omega \circ c) \chi_{\mathcal{S}}=\omega(1) \chi_{\mathcal{S}} \in \mathbf{C} \chi_{\mathcal{S}}
$$

It follows from the normality of $\rho$ that $\rho\left(L^{\infty}(K) \otimes \mathbf{C}\right) \chi_{\mathcal{S}} \subseteq \mathbf{C} p$. Hence the equation

$$
\rho(f \otimes 1) \chi_{\mathcal{S}}=\phi(f) \chi_{\mathcal{S}} \quad\left(f \in L^{\infty}(K)\right)
$$

defines a character $\phi$ on $L^{\infty}(K)$, which is by construction normal. This is possible exactly when $K$ is discrete, as any character on a non-atomic masa in $B(\mathcal{H})$ for some (separable) Hilbert space $\mathcal{H}$ must be singular (see [30, §10.19]). Thus we have proven the following.

PROPOSITION 8.1. Let $\alpha$ be a coaction of a locally compact group $K$ on a von Neumann algebra A having a Cartan subalgebra $D$ contained in $A^{\alpha}$. The group $K$ is discrete if and only if $\alpha$ is integrable.

COROLLARY 8.2. Let $\alpha$ be a coaction of a locally compact group $K$ on a von Neumann algebra A having a Cartan subalgebra $D$ contained in $A^{\alpha}$. If $\alpha$ is semidual, then $K$ is discrete.

Proof. Suppose that $\alpha$ is semidual. By [33, Proposition 5.12], $\alpha$ is integrable, and the homomorphism $\rho$ in Proposition 8.1 is faithful. Hence, by Proposition $8.1, K$ is discrete.

Finally, by making use of the Jones projection $\chi_{\mathcal{S}}$, we will give a complete answer when the surjection $\rho$ is bijective.

Proposition 8.3. Let the notation be as above. If $K$ is discrete, i.e. $\alpha=\alpha_{c}$ is integrable, the following assertions are equivalent:

(1) the surjective *-homomorphism $\rho$ is faithful;

(2) for each $k \in K, r\left(c^{-1}(k)\right)$ is a conull set;

(3) for each $k \in K$, there exists a countable set of normalizing groupoid $\left\{v_{k, i}\right\}_{i \in I_{k}}$ such that each $v_{k, i}$ belongs to $A^{\alpha}(k)$ and $\left\{v_{k, i} v_{k, i}^{*}\right\}_{i \in I_{k}}$ is a partition of unity.

Proof. (1) $\Rightarrow(2)$. Suppose that there exists $k \in K$ such that $\mu\left(X \backslash r\left(c^{-1}(k)\right)\right)>0$. Put $E:=X \backslash r\left(c^{-1}(k)\right)$ and $a:=L^{\sigma}\left(\chi_{E}\right)$. A direct computation shows that $\rho(\alpha(a)$ $\left.\left(\delta_{k} \otimes 1\right)\right)=0$, where $\delta_{k}$ is the function on $K$ defined by $\delta_{k}(m):=\delta_{k, m}$. So we conclude that $\rho$ is not faithful. 
(2) $\Rightarrow$ (3). This easily follows by using Zorn's lemma.

(3) $\Rightarrow(1)$. We claim that there exists the inverse to $\rho$. By using $\left\{v_{k, i}\right\}_{i \in I_{k}}$, we find that the projection $\chi_{c^{-1}(k)}$ coincides with $\sum_{i \in I_{k}} v_{k, i} \chi_{\mathcal{S}} v_{k, i}^{*}$. Let $a_{2} \in A_{2}$. For each $i \in I_{k}$, we have that $a_{2} v_{k, i} \chi_{\mathcal{S}} v_{k, i}^{*}$ is in $A v_{k, i} \chi_{\mathcal{S}} v_{k, i}^{*}$. So there exists a unique element $a_{k, i}$ in $A v_{k, i} v_{k, i}^{*}$ such that $a_{2} v_{k, i} \chi_{\mathcal{S}} v_{k, i}^{*}=a_{k, i} v_{k, i} \chi_{\mathcal{S}} v_{k, i}^{*}$. So we have

$$
a_{2}=\sum_{k \in K} \sum_{i \in I_{k}} a_{k, i} v_{k, i} \chi_{\mathcal{S}} v_{k, i}^{*}=\sum_{k \in K} \sum_{i \in I_{k}} a_{k, i} \chi_{c^{-1}(k)},
$$

in the sense of the strong operator topology. Let $\theta$ be the map from $A_{2}$ to $\widehat{K}_{\alpha} \ltimes A$ which is defined by the following:

$$
\theta\left(\sum_{k \in K} \sum_{i \in I_{k}} a_{k, i} \chi_{c^{-1}(k)}\right):=\sum_{k \in K} \sum_{i \in I_{k}} \alpha\left(a_{k, i}\right)\left(\delta_{k} \otimes 1\right) .
$$

Since $\sum_{i \in I_{k}} v_{k, i} v_{k, i}^{*}=1, \theta$ is well defined. Moreover, a direct computation shows that $\theta=\rho^{-1}$. In particular, $\rho$ is faithful.

\section{A. Appendix. Induced actions on von Neumann algebras}

This appendix is designed to collect together some fundamental facts about induced actions on von Neumann algebras. The arguments made in $\$ 7$ heavily rely on the theory of induced actions developed by Takesaki in [31]. As the readers might notice there, we have adopted a definition of the induced action that is slightly different to that of [31]. Although our definition is of course equivalent to Takesaki's, we feel that it is necessary for the sake of completeness of our arguments to state and prove the main results on induced actions in terms of our definition, which will be done in this Appendix A. Therefore, we have to emphasize that the two results obtained below are essentially nothing new.

Let $P$ be a von Neumann algebra. Also let $G$ be a locally compact group and $H$ a closed subgroup of $G$. Assume that we are given an action $\beta$ of $H$ on $P$. We consider $L^{\infty}(G) \otimes P$ and regard it as the set of all essentially bounded measurable $P$-valued functions on $G$. Define an action $\gamma$ of $H$ and an action $\kappa$ of $G$ on $L^{\infty}(G) \otimes P$ by

$$
\begin{gathered}
\gamma_{h}(X)(s):=\beta_{h}(X(s h)), \quad \kappa_{g}(X)(s):=X\left(g^{-1} s\right) \\
\left(X \in L^{\infty}(G) \otimes P, s, g \in G, h \in H\right) .
\end{gathered}
$$

Clearly, two actions $\gamma, \kappa$ commute. Put $Q:=\left(L^{\infty}(G) \otimes P\right)^{\gamma}$. For any $g \in G$, define $\alpha_{g}$ to be the restriction of $\kappa_{g}$ to $Q$. Thus we obtain an action $\alpha$ of $G$ on $Q$. This action $\alpha$ is called the action induced by $\beta$ [31]. The pair $\{Q, \alpha\}$ is usually denoted by $\operatorname{Ind}_{H}^{G}\{P, \beta\}$.

We denote by $\pi$ the canonical surjection from $G$ onto the quotient space $G / H$. Fix a quasi-invariant measure $\mu$ on $G / H$. Denote by $\rho$ the rho-function associated to this quasi-invariant measure (see [11, Section 2.6]). We also choose a Borel cross section $\theta: G / H \rightarrow G$ with $\theta(\pi(e))=e$. Then every $g \in G$ can be uniquely represented in the form $g=s_{g} h_{g}$, where $s_{g}=\theta(\pi(g))$. As in [4] and [38], we introduce a Borel 1-cocycle $\chi$ from $G \times(G / H)$ into $H$ by

$$
\chi(g, x):=h_{g \theta(x)}=\theta(g x)^{-1} g \theta(x) \quad(g \in G, x \in G / H) .
$$


Now we consider the von Neumann algebra $R:=L^{\infty}(G / H) \otimes P$ and regard it as the set of all essentially bounded measurable $P$-valued functions on $G / H$. Then we define an action $\delta$ of $G$ on $R$ by

$$
\delta_{g}(Y)(x)=\beta_{\chi\left(g^{-1}, x\right)}^{-1}\left(Y\left(g^{-1} x\right)\right) \quad(Y \in R, x \in G / H, g \in G) .
$$

By the cocycle identity of $\chi, \delta$ is indeed an action.

Proposition A.1. The induced action $\alpha$ of $G$ on $Q$ is conjugate to $\delta$ constructed above.

Proof. Define a map $\Psi$ from $R$ into $L^{\infty}(G) \otimes P$ by

$$
\Psi(Y)(g):=\beta_{h_{g}}^{-1}(Y(\pi(g))) \quad(Y \in R) .
$$

We then have

$$
\begin{aligned}
\gamma_{h}(\Psi(Y))(s) & =\beta_{h}(\Psi(Y)(s h))=\beta_{h}\left(\beta_{h_{s} h}^{-1}(Y(\pi(s)))\right) \\
& =\beta_{h_{s}}^{-1}(Y(\pi(s)))=\Psi(Y)(s) .
\end{aligned}
$$

This shows that $\Psi(Y)$ belongs to $Q$. Thus $\Psi$ maps $R$ into $Q$. It is easy to see that $\Psi$ is an injective $*$-homomorphism. In order to prove that $\Psi$ is surjective, introduce a map $\Phi$ from $Q$ into $R$ by

$$
\Phi(X)(x):=X(\theta(x)) \quad(X \in Q, x \in G / H) .
$$

Then we have

$$
\begin{aligned}
\Psi(\Phi(X))(g) & =\beta_{h_{g}}^{-1}(\Phi(X)(\pi(g)))=\beta_{h_{g}}^{-1}(X(\theta(\pi(g)))) \\
& =\beta_{h_{g}}^{-1}\left(X\left(s_{g}\right)\right)=\beta_{h_{g}}^{-1}\left(X\left(\left(s_{g} h_{g}\right) h_{g}^{-1}\right)\right) \\
& =\gamma_{h_{g}^{-1}}(X)(g)=X(g) .
\end{aligned}
$$

Hence $\Psi$ is surjective. Let $Y \in R$. Then we compute the following:

$$
\begin{aligned}
\Psi\left(\delta_{g_{0}}(Y)\right)(g) & =\beta_{h_{g}}^{-1}\left(\delta_{g_{0}}(Y)(\pi(g))\right)=\beta_{h_{g}}^{-1}\left(\beta_{\chi\left(g_{0}^{-1}, \pi(g)\right)}^{-1}\left(Y\left(g_{0}^{-1} \pi(g)\right)\right)\right) \\
& =\beta_{h_{g_{0}-1}^{-1} h_{g}}^{-1}\left(Y\left(\pi\left(g_{0}^{-1} g\right)\right)\right)=\beta_{h_{g_{0}}^{-1} g}^{-1}\left(Y\left(\pi\left(g_{0}^{-1} g\right)\right)\right) \\
& =\Psi(Y)\left(g_{0}^{-1} g\right)=\alpha_{g_{0}}(\Psi(Y))(g) .
\end{aligned}
$$

Therefore, $\alpha$ is conjugate to $\delta$.

THEOREM A.2. (Imprimitivity Theorem) Let $\alpha$ be an action of a locally compact group $G$ on a von Neumann algebra $Q$, and $H$ be a closed subgroup of $G$. Suppose that there exists a $G$-equivariant embedding of $L^{\infty}(G / H)$ into the center of $Q$. Then there exists an action $\beta$ of $H$ on a von Neumann algebra $P$ such that the induced action of $\beta$ is conjugate to $\alpha$.

Proof. In what follows, we fix a quasi-invariant measure $\mu$ on $G / H$. Choose a (separable) Hilbert space $\mathcal{H}$ so that $\{Q, \mathcal{H}\}$ is a standard representation. Let $U(g)$ be the canonical implementation of $\alpha_{g}$ on $\mathcal{H}$. So $U$ is a unitary representation of $G$ on $\mathcal{H}$. We identify $L^{\infty}(G / H)$ with the image $Z$ of the embedding above. Then we obtain the 
transitive imprimitivity system for $\{U, \mathcal{H}\}$. By the Mackey-Blattner theorem for induced representations, there exists a unitary representation $\{L, \mathcal{K}\}$ of $H$ such that $U$ is the induced representation of $L$. Therefore, $\mathcal{H}$ can be identified with $L^{2}(G / H, \mu) \otimes \mathcal{K}=L^{2}(G / H, \mathcal{K})$. Then $U$ can be expressed in the form

$$
\begin{aligned}
\left\{U\left(g_{0}\right) \eta\right\}(x)= & {\left[\frac{d\left(\mu \circ g_{0}^{-1}\right)}{d \mu}(x)\right]^{1 / 2} L_{\chi\left(g_{0}^{-1}, x\right)}^{-1} \eta\left(g_{0}^{-1} x\right) } \\
& \left(\eta \in L^{2}(G / H, \mathcal{K}), g_{0} \in G, x \in G / H\right),
\end{aligned}
$$

where $\chi: G \times G / H \rightarrow H$ is the 1-cocycle defined earlier.

To any $Y \in Z^{\prime}$, there corresponds an essentially bounded $B(\mathcal{K})$-valued function $Y(\cdot)$ on $G / H$ such that $\{Y \eta\}(x)=Y(x) \eta(x)$ for all $\eta \in L^{2}(G / H, \mathcal{K})$ [32, Theorem 7.10]. Following the notation in [32], we write $Y=\int_{G / H}^{\oplus} Y(x) d \mu(x)$ in this case. If $Y=$ $\int_{G / H}^{\oplus} Y(x) d \mu(x) \in Q$, then we have

$$
\alpha_{g}(Y)=\int_{G / H}^{\oplus} Y\left(g^{-1} x\right) d \mu(x) .
$$

Let $A$ be the set of all $Y \in Q$ such that $\lim _{g \rightarrow e}\left\|\alpha_{g}(Y)-Y\right\|=0$. If $Y$ has the form $Y=\int_{G / H}^{\oplus} Y(x) d \mu(x)$, then the limit can be rewritten as $\lim _{g \rightarrow e}\left\|Y\left(g^{-1} \cdot\right)-Y(\cdot)\right\|_{\infty}=0$. Let $Y=\int_{G / H}^{\oplus} Y(x) d \mu(x) \in A$. Then, for each $\omega \in B(\mathcal{K})_{*}, g \in G \mapsto\langle Y(\pi(g)), \omega\rangle \in \mathbf{C}$ is essentially bounded. Take a strong lifting $\varrho$ of $L^{\infty}(G)$ that commutes with the left translation [18]. Let $F_{Y, \omega}:=\varrho(\langle Y(\pi(\cdot)), \omega\rangle)$. From the property of $Y(\cdot)$, it follows that $F_{Y, \omega}$ is uniformly continuous. For each $g \in G$, there exists a unique element $S_{Y}(g) \in B(\mathcal{K})$ such that $\left\langle S_{Y}(g), \omega\right\rangle=F_{Y, \omega}(g)$ for all $\omega \in B(\mathcal{K})_{*}$. Let $P$ be the von Neumann subalgebra of $B(\mathcal{K})$ generated by $\left\{S_{Y}(e): Y \in Q\right\}$. Let $\tilde{Y}(x):=S_{Y}(\theta(x))$ for all $x \in G / H$. It is then easy to check that $\tilde{Y}(x)=Y(x)$ for $\mu$-almost every $x \in G / H$. So $\tilde{Y}:=\int_{G / H}^{\oplus} \tilde{Y}(x) d \mu(x)=Y$. Let $g_{0} \in G$ and $Y \in A$. Then $\alpha_{g_{0}}(\tilde{Y})=\alpha_{g_{0}}(Y)$ is in $A$. For any $\omega \in B(\mathcal{K})_{*}$, since the lifting $\rho$ commutes with the left translation, we have

$$
\begin{aligned}
F_{\alpha_{g_{0}}(\tilde{Y}), \omega} & =\varrho\left(\left\langle\alpha_{g_{0}}(\tilde{Y})(\pi(\cdot)), \omega\right\rangle\right)=\varrho\left(\left\langle\tilde{Y}\left(g_{0}^{-1} \pi(\cdot)\right), \omega\right\rangle\right) \\
& =\varrho\left(\left\langle\tilde{Y}\left(\pi\left(g_{0}^{-1} \cdot\right)\right), \omega\right\rangle\right)=F_{\tilde{Y}, \omega}\left(g_{0}^{-1} \cdot\right) .
\end{aligned}
$$

This yields

$$
S_{\alpha_{g_{0}}(Y)}(g)=S_{\alpha_{g_{0}}(\tilde{Y})}(g)=S_{\tilde{Y}}\left(g_{0}^{-1} g\right)=S_{Y}\left(g_{0}^{-1} g\right) .
$$

In particular, $\widetilde{\alpha_{g_{0}}(\tilde{Y})}(x)=S_{\alpha_{g_{0}}(\tilde{Y})}(\theta(x))=S_{Y}\left(g_{0}^{-1} \theta(x)\right)$ and $S_{Y}\left(g_{0}^{-1}\right)=S_{\alpha_{g_{0}}(Y)}(e) \in P$.

Let $h \in H$ and $Y \in A$. For any $\xi \in \mathcal{K}$ and any continuous function $\phi$ on $G / H$ with compact support, we have

$$
\begin{aligned}
\phi(\pi(e)) L_{h} S_{Y}(e) L_{h}^{*} \xi & =\left[\frac{d(\mu \circ h)}{d \mu}(\pi(e))\right]^{-1 / 2} L_{h} \tilde{Y}(\pi(e))\left\{U(h)^{*}(\phi \otimes \xi)\right\}(\pi(e)) \\
& =\left[\frac{d(\mu \circ h)}{d \mu}(\pi(e))\right]^{-1 / 2} L_{h}\left\{\tilde{Y} U(h)^{*}(\phi \otimes \xi)\right\}(\pi(e)) \\
& =\left\{U(h) \tilde{Y} U(h)^{*}(\phi \otimes \xi)\right\}(\pi(e)) \\
& =\left\{\alpha_{h}(\tilde{Y})(\phi \otimes \xi)\right\}(\pi(e))=\phi(\pi(e)) \alpha_{h}(\tilde{Y})(\pi(e)) \xi \\
& =\phi(\pi(e)) S_{\alpha_{h}(Y)}(e) \xi
\end{aligned}
$$


Hence we obtain $L_{h} S_{Y}(e) L_{h}^{*}=S_{\alpha_{h}(Y)}(e) \in P$. It follows that $\left.\operatorname{Ad} L_{h}\right|_{P}$ defines an action $\beta$ of $H$ on $P$. From the way we constructed the action $\beta$, it is plain to see that the induced action of $\beta$ is the original action $\alpha$.

\section{REFERENCES}

[1] S. Adams, G. A. Elliott and T. Giordano. Amenable actions of groups. Trans. Amer. Math. Soc. 344 (1994), 803-822.

[2] H. Aoi. A construction of equivalence subrelations for intermediate subalgebras. J. Math. Soc. Japan 55 (2003), 713-725.

[3] H. Aoi and T. Yamanouchi. Construction of a canonical subfactor for an inclusion of factors with a common Cartan subalgebra. Hokkaido Math. J. 32 (2003), 41-58.

[4] A. O. Barut and R. Raczka. Theory of Group Representations and Applications. World Scientific, Singapore, 1986.

[5] A. Connes, J. Feldman and B. Weiss. An amenable equivalence relation is generated by a single transformation. Ergod. Th. \& Dynam. Sys. 1 (1981), 431-450.

[6] M. Enock. Sous-facteurs intermediaires et groupes quantiques mesures. J. Operator Theory 42 (1999), 305-330.

[7] P. Eymard. L'algèbre de Fourier d'un groupe localement compact. Bull. Soc. Math. France 92 (1964), 181-236.

[8] J. Feldman, P. Hahn and C. Moore, Orbit structure and countable sections for actions of continuous groups. Adv. Math. 28 (1978), 186-230.

[9] J. Feldman and C. C. Moore, Ergodic equivalence relations, cohomology, and von Neumann algebras, I. Trans. Amer. Math. Soc. 234 (1977), 289-324.

[10] J. Feldman and C. C. Moore. Ergodic equivalence relations, cohomology, and von Neumann algebras, II. Trans. Amer. Math. Soc. 234 (1977), 325-359.

[11] G. B. Folland. A Course in Abstract Harmonic Analysis. CRC Press, Boca Raton, FL, 1995.

[12] A. Furman. Orbit equivalence rigidity. Ann. Math. 150 (1999), 1083-1108.

[13] V. Ya. Golodets and S. D. Sinel'shchikov. Outer conjugacy for actions of continuous amenable groups. Publ. Res. Inst. Math. Sci. Kyoto Univ. 23 (1987), 737-769.

[14] V. Ya. Golodets and S.D. Sinel'shchikov. Classification and structure of cocycles of amenable ergodic equivalence relations. J. Funct. Anal. 121 (1994), 455-485.

[15] P. Hahn. The regular representations of measure groupoids. Trans. Amer. Math. Soc. 242 (1978), 35-72.

[16] U. Haagerup and E. Størmer, Equivalence of normal states on von Neumann algebras and the flow of weights. Adv. Math. 83 (1990), 180-262.

[17] M. Izumi, R. Longo and S. Popa. A Galois correspondence for compact groups of automorphisms of von Neumann algebras with a generalization to Kac algebras. J. Funct. Anal. 155 (1998), 25-63.

[18] A. Ionescu Tulcea and C. Ionescu Tulcea. On the existence of a lifting commuting with the left translations of an arbitrary locally compact group. Proc. Fifth Berkeley Symp. on Mathematical Statistics and Probability. Vol. II, Part 1. University of California Press, California, 1967, pp. 63-97.

[19] Y. Kawahigashi. One-parameter automorphism groups of the hyperfinite type $\mathrm{II}_{1}$ factor. J. Operator Theory 25 (1991), 37-59.

[20] J. Kustermans and S. Vaes. Locally compact quantum groups in the von Neumann algebraic setting. Math. Scand. 92 (2003), 68-92.

[21] Y. Nakagami. Dual action on a von Neumann algebra and Takesaki's duality for a locally compact group. Publ. Res. Inst. Math. Sci. Kyoto Univ. 12 (1977), 727-775.

[22] Y. Nakagami. Essential spectrum $\Gamma(\beta)$ of a dual action on a von Neumann algebra. Pacific J. Math. 70 (1977), 437-479.

[23] Y. Nakagami and M. Takesaki. Duality for Crossed Products of von Neumann Algebras (Lecture Notes in Mathematics, 731). Springer, Berlin, 1979.

[24] A. Ramsay. Topologies on measured groupoids. J. Funct. Anal. 47 (1982), 314-343.

[25] J. Renault. A Groupoid Approach to $C^{*}$-algebras (Lecture Notes in Mathematics, 793). Springer, Berlin, 1980. 
[26] H.L. Royden. Real Analysis. Prentice-Hall, Englewood Cliffs, NJ, 1988.

[27] K. Schmidt. Cocycles on Ergodic Transformation Groups. MacMillan Company of India, Ltd., Delhi, 1977.

[28] K. Schmidt. Algebraic Ideas in Ergodic Theory (Conference Board of Mathematical Sciences Regional Conference Series in Mathematics, 76). American Mathematical Society, Washington, DC, 1990.

[29] Y. Sekine. An analogue of Paschke's theorem for actions of compact Kac algebras. Kyushu J. Math. 52 (1998), 353-359.

[30] S. Strătilă. Modular Theory in Operator Algebras. Abacus Press, Tunbridge Wells, 1981.

[31] M. Takesaki. Duality for crossed products and the structure of von Neumann algebras of type III. Acta Math. 131 (1973), 249-310.

[32] M. Takesaki. Theory of Operator Algebras I. Springer, New York, 1979.

[33] S. Vaes. The unitary implementation of a locally compact quantum group action. J. Funct. Anal. 180 (2001), 426-480.

[34] T. Yamanouchi. Crossed products by groupoid actions and their smooth flows of weights. Publ. RIMS, Kyoto Univ. 28 (1992), 535-578.

[35] T. Yamanouchi. Dual weights on crossed products by groupoid actions. Publ. RIMS, Kyoto Univ. 28 (1992), 653-678.

[36] T. Yamanouchi. Galois groups of quantum group actions and regularity of fixed-point algebras. Trans. Amer. Math. Soc. 355 (2003), 2813-2828.

[37] R. J. Zimmer. Extension of ergodic group actions. Illinois J. Math. 20 (1976), 373-409.

[38] R. J. Zimmer. Induced and amenable ergodic actions of Lie groups. Ann. Sci. École. Norm. Sup. (4) série, 11 (1978), 407-428. 